\title{
Fine-structure constant variability, equivalence principle and cosmology
}

\author{
Jacob D. Bekenstein* \\ Racah Institute of Physics, Hebrew University of Jerusalem \\ Givat Ram, Jerusalem 91904 ISRAEL
}

(Dated: October 23, 2018)

\begin{abstract}
It has been widely believed that variability of the fine-structure constant $\alpha$ would imply detectable violations of the weak equivalence principle. This belief is not justified in general. It is put to rest here in the context of the general framework for $\alpha$ variability [J. D. Bekenstein, Phys. Rev. D 25, 1527 (1982)] in which the exponent of a scalar field plays the role of the permittivity and inverse permeability of the vacuum. The coupling of particles to the scalar field is necessarily such that the anomalous force acting on a charged particle by virtue of its mass's dependence on the scalar field is cancelled by terms modifying the usual Coulomb force. As a consequence a particle's acceleration in external fields depends only on its charge to mass ratio, in accordance with the principle. And the center of mass acceleration of a composite object can be proved to be independent of the object's internal constitution, as the weak equivalence principle requires. Likewise the widely employed assumption that the Coulomb energy of matter is the principal source of the scalar field proves wrong; Coulomb energy effectively cancels out in the continuum description of the scalar field's dynamics. This cancellation resolves a cosmological conundrum: with Coulomb energy as the source of the scalar field, the framework would predict a decrease of $\alpha$ with cosmological expansion, whereas an increase is claimed to be observed. Because of the said cancellation, magnetic energy of cosmological baryonic matter is the main source of the scalar field. Consequently the expansion is accompanied by an increase in $\alpha$; for reasonable values of the framework's sole parameter, this occurs at a rate consistent with the observers' claims.
\end{abstract}

\section{INTRODUCTION}

Observations of fine structure multiplet splittings in the absorption line systems of distant quasars have lately suggested [1] that the fine-structure constant $\alpha$ at cosmological epochs with redshift 1-3.5 was lower than it is today. This evidently revolutionary claim begs for a theoretical framework to enable judgment as to whether such a variation can be consistent with accepted physical principles. Variation of $\alpha$ was first considered theoretically by Jordan [2], Teller [3] and Stanyukovich [4].

Already before Gamow's influential speculation that $\alpha$ varies linearly with cosmological time [5], Dicke had made the point that any variation of $\alpha$ can be regarded equally well as due to variation of particle charge, or alternatively of the speed of light $c$ or of $\hbar$, with the choice being a matter of convenience, not physics [6]. He also exhibited a theory in which the Maxwell invariant couples linearly to a standard massless scalar field as an example of a variable $\alpha$ theory. In this theory the permittivity and reciprocal permeability of the vacuum vary in consonance with the scalar field; it can also be regarded as a variable charge theory. Independently of the specific theory of $\alpha$ variation, Dicke provided an argument [6,7] that spatial variation of $\alpha$, which would be expected to accompany cosmological temporal variation if the underlying theory is covariant, contradicts the weak equivalence principle (WEP). The essence of the argument is that a non-negligible fraction $\zeta$ of the mass $M$ of any chunk of ordinary neutral matter is Coulombic in origin. The Coulomb energy $E_{C}=\zeta M c^{2}$ should scale with the square of the constituent charges, meaning it should be proportional to $\alpha$, and should thus depend on position if $\alpha$ does. The force on mass $M$ should thus contain in addition to the Newtonian part $-M \boldsymbol{\nabla} \phi_{N}$ an anomalous portion $-\boldsymbol{\nabla} E_{C}=-\left(\partial E_{C} / \partial \alpha\right) \boldsymbol{\nabla} \alpha=-\left(\zeta M c^{2} / \alpha\right) \boldsymbol{\nabla} \alpha$. Dicke conjectured [6, 7] that $\nabla \alpha / \alpha \approx \alpha c^{-2} \nabla \phi_{N}$. In this view - here designated the Coulomb model regardless of the law of $\alpha$ variation assumed - the acceleration of $M$ comprises an anomalous fraction $\zeta \alpha$ which varies from material to material (for example, the nuclear contribution to $\zeta$ ranges from 0.0016 for aluminum to 0.0041 for lead), thus engendering a violation of the principle of universality of free fall of neutral matter, a special case of the WEP. Uzan [8] has given a masterly review of this subject, as well as of the whole question of $\alpha$ variability.

I made use of the Coulomb model for the anomalous force when drawing conclusions from the general field-theoretic framework of $\alpha$ variability I formulated two decades ago [9]. The model has also been uncritically adopted by most subsequent investigations in the subject $[10,11,12,13,14,15,16,17,18]$; a refreshing exception is Landau et al [19].

*Electronic address: bekenste@vms.huji.ac.il; URL: http://www.phys.huji.ac.il/bekenste/ 
Within the general framework the Coulomb model predicts that the anomalous acceleration is a fraction $\sim \zeta^{2}$ of the total one; this is within an order of magnitude of the fraction $\zeta \alpha$ implied by Dicke's conjecture (see Ref. [9] and Sec. V A below). Similar results are in evidence in other treatments. They have given rise to the widespread belief that $\alpha$ variability necessarily implies violations of the WEP detectable by Eötvös-Dicke-Braginsky (EDB) type experiments [8], particularly if the claimed cosmological variability [1] is essentially correct.

But as shown below, such sweeping conclusion is unwarranted; it all depends on the structure of the underlying field theory. Contrary to intuition and my original supposition, in the general framework [9] Coulomb energy of matter is found to be unimportant as a source of the scalar field responsible for spatial $\alpha$ variability. Spatial gradients of that field are necessarily much smaller than has been generally appreciated heretofore. This finding also impinges upon cosmology as it leads to a modification of the widely used cosmological equation for $\alpha$ variability.

The paper is designed as follows. In Sec. II I recapitulate the general variable $\alpha$ framework and discuss its relation to other theories of $\alpha$ variability. I rederive the equations of motion for scalar and electromagnetic fields and particles within the framework, and remark that one is prevented from assuming — as widely done — that the dependence of particle masses on the scalar field, which enters both in the anomalous force on particles and in the source of scalar field, comes from Coulomb energy. Rather the equations of the theory themselves, being nonlinear, determine the nature of their sources.

This is indeed seen from the exact solution - presented in Sec. III — for the fields of a pointlike charge held initially at rest in a uniform external electric field. The dependence of the particle's mass on scalar field is not of the sort expected from the Coulomb energy model: the anomalous force is cancelled by a correction to the usual electric force. Thus there is no indication that different types of particle with like charge-to-mass ratio would move differently in the same external fields, and so there is no basis for a violation of the WEP. I also show that within the framework a charged particle has a minimum possible extension, and that gravitational corrections to the mentioned results are small, even for the most compact charge.

In Sec. IV I generalize the said solution to the case of many charges held fixed in space. I prove rigorously that at an initial moment, the center-of-mass acceleration of a collection of charges (some may be zero) starting at rest depends exclusively on its total mass and charge, and on the external electric field, but not on the structure of the collection. Again no violation of the WEP is in evidence.

Sec. V shifts the focus from the microscopic description of individual charges to the macroscopic description in terms of smoothed electromagnetic and scalar fields and their smoothed sources. The macroscopic scalar field is shown by a variety of examples to be too small to effect WEP violations detectable in the foreseeable future. Ordinary matter contributes two terms of Coulomb origin to the source of the scalar field. Taken together uncritically these would suggest that in the vicinity of a chunk of matter, $\nabla \alpha / \alpha \sim \zeta c^{-2} \nabla \phi_{N}$, of the same order as Dicke's conjectured spatial $\alpha$ variability. But when the mentioned exact solution is taken into account, the two Coulomb terms are seen to cancel each other. The source retains only terms of higher order; these are incapable of generating WEP violating signals observable in the foreseeable future.

Casting about for other sources of $\alpha$ variability, I show in Sec. VI that contributions of spin and orbital magnetic dipoles in ordinary matter to the sources of the scalar field are likewise too weak to engender violations of the WEP at soon-to-be observable levels.

In Sec. VII I show that the cancellation of the Coulomb energy in the source of the scalar field makes baryonic magnetic energy the dominant source of cosmological $\alpha$ variability. This, together with the significant value of the fundamental scale of the theory permitted by WEP tests (as newly understood), makes it possible to understand the observed cosmological growth of $\alpha$ and the rate of it as reflecting standard properties of cosmological baryonic matter; there is no need for this to postulate dark matter with peculiar electromagnetic properties. Sec. VIII summarizes the conclusions and caveats on them.

Below I shall employ the signature $\{-1,1,1,1\}$ and the convention that Greek indices range from 0 to 3 , while Latin indices take on values from 1 to 3 . The time coordinate is denoted by $t$ or $x^{0}$.

\section{FRAMEWORK FOR $\alpha$ VARIABILITY}

\section{A. Modifying electrodynamics}

The general field-theoretic framework for $\alpha$ variability [9] is based on eight assumptions: (1) for constant $\alpha$ the framework's electromagnetism reduces to Maxwell's with a minimal coupling to charged matter, (2) $\alpha$ dynamics comes from an action, (3) this as well as electrodynamics' action are relativistic invariants, (4) the overall action respects gauge invariance, (5) electromagnetism is causal and (6) respects time reversal-invariance, (7) any length scale in the theory is not smaller than Planck's length $\ell_{P}=\left(\hbar G / c^{3}\right)^{1 / 2} \approx 1.616 \times 10^{-33} \mathrm{~cm}$, and (8) gravitation is governed by the Einstein-Hilbert action. 
The choice of units that makes $G, \hbar$ and $c$ constant shifts the burden of variation onto the charges. Simplest is the case where all charges $e_{i}$ vary in unison: $e_{i}=e_{0 i} \epsilon\left(x^{\mu}\right)$, where $e_{0 i}$ denotes the coupling constant of particle $i$ and $\epsilon\left(x^{\mu}\right)$ is a dimensionless scalar field (scalar since charge is an invariant in relativity). There is arbitrariness in the definition of $\epsilon$; one can multiply it by a constant and divide all $e_{0 i}$ by the same constant without changing anything. That is why one must demand that the dynamics of $\epsilon$ be invariant under global rescaling of this field (charge-scale invariance). The only possible form of the free action for $\epsilon$ is thus

$$
S_{\epsilon}=-\frac{\hbar c}{2 l^{2}} \int \epsilon^{-2} \epsilon_{, \mu} \epsilon^{\mu}(-g)^{1 / 2} d^{4} x,
$$

where $g$ denotes the determinant of the metric $g_{\mu \nu}, l$ is a constant scale of length introduced for dimensional reasons; by assumption $l$ cannot be smaller than $\ell_{P}$.

By assumption (1) $\epsilon$ must enter into all electromagnetic interaction terms in the matter action via the replacement $e_{i} A_{\mu} \mapsto e_{0 i} \epsilon A_{\mu}$, with $A_{\mu}$ the usual electromagnetic potential. Gauge invariance of the matter action [assumption (4)] will then be preserved only if a gauge transformation means

$$
\epsilon A_{\mu} \mapsto \epsilon A_{\mu}+\lambda_{, \mu}
$$

with $\lambda$ any scalar function of spacetime point. In order for the electromagnetic action to be invariant under this transformation as well as under $\epsilon$ rescaling, it must take the form

$$
S_{\mathrm{em}}=-\frac{1}{16 \pi} \int F_{\mu \nu} F^{\mu \nu}(-g)^{1 / 2} d^{4} x,
$$

where $F_{\mu \nu}=\epsilon^{-1}\left[\left(\epsilon A_{\nu}\right)_{, \mu}-\left(\epsilon A_{\mu}\right)_{, \nu}\right]$ obviously stands for the gauge and $\epsilon$-scale invariant electromagnetic field tensor. One can consider adding to the integrand of $S_{\text {em }}$ a term such as $F_{\mu \nu}{ }^{*} F^{\mu \nu}$, where ${ }^{*} F^{\mu \nu}$ denotes the dual of $F_{\mu \nu}$. In Maxwellian electrodynamics such an addition is equivalent to a boundary term, and classically irrelevant. Here this is not true because of the appearance of the factor $\epsilon^{-2}$ in the integrand, but the extra term must nevertheless be rejected because it violates time-reversal invariance [assumption (6)].

\section{B. Actions}

The appearance of $\epsilon$ in the electromagnetic interaction means that the equation for $\epsilon$ will involve $A_{\mu}$. This was found to be a bit inconvenient in Ref. [9]. Thus I shall here adopt the Sandvik-Barrow-Magueijo (SBM) procedure [14] of replacing $A_{\mu}$ by another 4-potential, $a_{\mu} \equiv \epsilon A_{\mu}$. The gauge transformation (2) now turns into

$$
a_{\mu} \mapsto a_{\mu}+\lambda_{, \mu}
$$

so that it is suitable to think of

$$
f_{\mu \nu} \equiv a_{\nu, \mu}-a_{\mu, \nu}
$$

as the new electromagnetic field tensor; it will turn out to be the physical field tensor (Sec. II D). Likewise I express $\epsilon$ everywhere in terms of SBM's field $\psi=\ln \epsilon$.

The total action thus becomes $S=S_{(\psi)}+S_{(f)}+S_{\mathrm{m}}+S_{\mathrm{g}}$, where $S_{\mathrm{m}}$ is the matter action (including the electromagnetic interaction)

$$
\begin{aligned}
S_{(\psi)} & =-\frac{1}{8 \pi \kappa^{2}} \int \psi_{, \mu} \psi_{,}^{\mu}(-g)^{1 / 2} d^{4} x \\
S_{(f)} & =-\frac{1}{16 \pi} \int e^{-2 \psi} f_{\mu \nu} f^{\mu \nu}(-g)^{1 / 2} d^{4} x \\
S_{\mathrm{g}} & =\frac{c^{4}}{16 \pi G} \int R(-g)^{1 / 2} d^{4} x
\end{aligned}
$$

with

$$
\kappa \equiv \frac{l}{(4 \pi \hbar c)^{1 / 2}} \approx 8.11 \times 10^{-26} \frac{l}{\ell_{P}} \frac{\mathrm{erg}^{1 / 2}}{\mathrm{~cm}^{1 / 2}}
$$


In the new form of $S, \psi$ enters not only in $S_{(f)}$ but also appears in the particle masses in $S_{\mathrm{m}}$, even for elementary particles. This point is clear from the example of a fermion particle coupled to electromagnetism. If charges were truly constant, the process of renormalization would introduce $e_{i}$ dependence in the fermion (dressed) mass. After the transition

$$
e_{i} A_{\mu} \mapsto e_{0 i} \epsilon A_{\mu}
$$

the mass becomes a function of $\epsilon$. Swapping $a_{\mu}$ for $A_{\mu}$ eliminates $\epsilon$ from the electromagnetic interaction, but leaves $\epsilon$ or $\psi$ dependence in the mass. It is intractable to calculate such dependence by summing the quantum corrections to all orders in perturbation theory. However, the framework offers a self-consistent way to compute $m(\psi)$ which is investigated in Appendix C.

\section{Relation to other theories}

As discussed in Ref. [9] and already foreseen by Dicke (Ref. [6], Appendix 4), the second form of the framework describes constant charges in the presence of varying vacuum permittivity and permeability, $e^{-2 \psi}$ and $e^{2 \psi}$, respectively. The framework's action differs from that in Dicke's theory only in that here the electromagnetic Lagrangian is coupled to an exponential of the scalar field (as required by charge-scale invariance of the original form of the framework) whereas Dicke made the coupling linear in the field. There is a resemblance also to Jordan's theory of varying natural constants $[2,8]$; apart from the coefficients of the various terms, the action recapitulated here is the case $\eta=0$ of Jordan's.

This is the place to mention the variable speed-of-light (VSL) theories [20] which have also been touted as variable $\alpha$ theories [13, 21]. Dicke's dictum [6] (c.f. Sec. I) that only variation of a dimensionless constant is operationally meaningful does not preclude the formulation of a theory which promotes $e$, or alternatively $c$, to the status of a dynamical field. It only asserts that when $e$ and $c$ appear in the same physical context, the two forms of the theory would be experimentally indistinguishable [22, 23]. This point has led to loud controversy with some authors affirming that a VSL theory makes different predictions for $\alpha$ variability than a variable $e$ theory [13, 21, 24], while others deny it $[23]$.

This disagreement is easily defused; $c$ appears in physical actions in at least four contexts: in the electromagnetic field-to-matter coupling, cf. Eq. (11), in mass terms, in Lagrangian prefactors, e.g. the $\hbar c$ prefacing the Dirac field action, and in the Einstein-Hilbert action (8). Only for the first of these is $c$ variation fully swappable for $e$ 's in the context of variable $\alpha$. In fact, the variable factor in the $c$ featuring in the electromagnetic field-to-matter coupling could be absorbed into the electromagnetic potential at the sole cost of introducing a dynamical factor in the electromagnetic field action (3), which would then take a form reminiscent of $S_{f}$, Eq. (7) of the framework studied here (a variable $e$ theory). However, a theory where at least two $c$ 's with different roles are promoted to different dynamical fields can obviously make predictions different from those of a theory where only e varies. Such, for example, is Magueijo's covariant VLS [21]. In the case that all matter is just electromagnetic field, this theory can be understood as one where the $c$ of electromagnetism and that from the gravitational action are promoted to different powers of the scalar field. This theory, which essentially coincides with the general case of Jordan's [2, 8], would predict a different cosmological $\alpha$ evolution than does the present framework [13]. But that is because the modified Maxwellian electrodynamics in Magueijo's theory is supplemented by a Brans-Dicke style modification of gravity: it is a theory of variable $\alpha$ and variable gravitational coupling.

\section{Equations of motion}

Just as in other theories, in the framework the simplest $S_{\mathrm{m}}$ is that describing a pointlike classical, possibly charged, particle:

$$
S_{\mathrm{m}}=\int\left[-m c\left(-g_{\mu \nu} \frac{d z^{\mu}}{d \tau} \frac{d z^{\nu}}{d \tau}\right)^{1 / 2}+\frac{e_{0}}{c} \frac{d z^{\mu}}{d \tau} a_{\mu}\right] c \gamma^{-1} \delta^{3}[\mathbf{x}-\mathbf{z}(\tau)] d^{4} x
$$

where $z^{\mu}(\tau)=\left\{z^{0}(\tau), \mathbf{z}(\tau)\right\}$ is the world line of the particle as function of proper time $\tau$, and $\gamma=d z^{0} / d \tau$ is its Lorentz factor. The vanishing of $\delta S_{\mathrm{m}} / \delta x^{\mu}$, when combined with the condition $g_{\mu \nu} d z^{\mu} d z^{\nu}=-c^{2} d \tau^{2}$, gives the equation of motion

$$
\frac{D\left(m u_{\alpha}\right)}{d \tau}=-\frac{\partial m c^{2}}{\partial \psi} \psi, \alpha+\frac{e_{0}}{c} f_{\alpha \beta} u^{\beta}
$$


or

$$
m \frac{D u_{\alpha}}{d \tau}=-\frac{\partial m c^{2}}{\partial \psi}\left(\psi, \alpha+u_{\alpha} u^{\beta} \psi_{, \beta}\right)+\frac{e_{0}}{c} f_{\alpha \beta} u^{\beta}
$$

where $D / d \tau$ stands for the covariant derivative along the velocity $u^{\alpha} \equiv d z^{\alpha} / d \tau$, itself subject to $g_{\mu \nu} u^{\mu} u^{\nu}=-c^{2}$. It is plain from this that $f_{\alpha \beta}$ is the physical electromagnetic field. Eq. (13) makes it clear that in general, in addition to the (suitably modified) Coulomb and Lorentz forces, an anomalous force coming from the $\psi$ dependence of mass acts on any test charge immersed in a background electromagnetic field.

The vanishing of $\delta\left(S_{\mathrm{m}}+S_{(f)}\right) / \delta a_{\mu}$ gives the electromagnetic field equations

$$
\begin{aligned}
\left(e^{-2 \psi} f^{\mu \nu}\right)_{; \nu} & =\frac{4 \pi}{c} j^{\mu} \\
j^{\mu} & \equiv e_{0} c u^{\mu} \frac{\delta^{3}[\mathbf{x}-\mathbf{z}(\tau)]}{\gamma \sqrt{ }-g}
\end{aligned}
$$

The appearance of $e^{-2 \psi}$ in Eq. (14) confirms the interpretation of this factor as permittivity (or reciprocal of the permeability) of the vacuum in the present version of the theory. The conservation of the current $j^{\mu}$ follows directly from Eq. (14); the conserved charge is the truly constant $e_{0}$. It is in this sense that electric charge is still conserved in this "variable charge framework" [9].

Finally the vanishing of $\delta\left(S_{\mathrm{m}}+S_{(\psi)}+S_{(f)}\right) / \delta \psi$ gives the equation for $\psi$,

$$
\psi_{, \mu}{ }^{\mu}{ }^{2}=4 \pi \kappa^{2} \frac{\partial m c^{3}}{\partial \psi} \frac{\delta^{3}[\mathbf{x}-\mathbf{z}(\tau)]}{\gamma \sqrt{ }-g}-\frac{\kappa^{2}}{2} e^{-2 \psi} f_{\mu \nu} f^{\mu \nu}
$$

while that of $\delta\left(S_{\mathrm{m}}+S_{(\psi)}+S_{(f)}+S_{\mathrm{g}}\right) / \delta g^{\mu \nu}$ gives the gravitational field equations.

To fully specify the equation of motion of charged particles, Eq. (13), one must specify $m(\psi)$. As mentioned in Sec. I, it has been customary to assume that the spacetime dependence of mass reflects the electromagnetic contribution to it, here proportional to $\epsilon^{2}$ or $e^{2 \psi}$. Thus for ordinary matter (for which the Coulomb energy far surpasses magnetic energy), it has been customarily assumed that a fixed fraction $\zeta$ of $m c^{2}$ is Coulombic, so that $\partial m / \partial \psi=2 \zeta m c^{2}[9,10,11,12,13,14,15,16,17,18]$. Because $\zeta$ is expected to vary from object to object, violation of the WEP would seem to be inevitable. However — and this is one of the main points of the present paper the mentioned prescription for $m(\psi)$ is the wrong one for the framework defined in Sec. II. The point is that the field equations (14)-(16) are nonlinear, e.g. the permittivity $e^{-2 \psi}$ is determined by the electromagnetic field strength through Eq. (16). It is well known that the equations of motion of the sources of nonlinear field equations, e.g. Einstein's equations, cannot be freely prescribed. Rather, their nature is specified by the field equations themselves. Here, too, one must let the field equations specify the nature of $m(\psi)$ which defines the explicit form of the source's equation of motion, Eq. (13).

\section{ISOLATED POINT ELECTRIC CHARGE}

\section{A. The charge's fields}

An important step in clearing up the status of the WEP in the framework is the study of the motion of a pointlike charge in a specified external electric field. The theory's nonlinearity does not permit one to ignore the charge's own fields in setting up its equation of motion; the task would be easier in Maxwellian electrodynamics where fields can be superposed. For now I neglect the curvature of spacetime; as shown in Sec. IIID, this is entirely justified for elementary particles and small collections of them. I also assume that initially the charge is held in place by some unspecified force, and then released, so that its fields are static to start with.

Setting $\gamma=1$ and $\mathbf{x}(\tau)=0$ in Eqs. (14)-(16) one can look for a static solution with vanishing magnetic field $\left(f^{i j}=0\right)$. With the notation $c^{-1} \mathbf{E} \equiv\left\{f^{01}, f^{02}, f^{03}\right\}$ one gets

$$
\begin{aligned}
\boldsymbol{\nabla} \cdot\left(e^{-2 \psi} \mathbf{E}\right) & =4 \pi e_{0} \delta^{3}(\mathbf{x}) \\
\nabla^{2} \psi & =4 \pi \kappa^{2}\left[\frac{\partial m c^{2}}{\partial \psi} \delta^{3}(\mathbf{x})+\frac{1}{4 \pi} e^{-2 \psi} \mathbf{E}^{2}\right]
\end{aligned}
$$

By Eq. (5) and the obvious condition that $a_{\mu}$ may be taken time independent, $\mathbf{E}$ must be a gradient: $\mathbf{E}=\nabla \Upsilon$. 
The generic solution of Eq. (17) is

$$
e^{-2 \psi} \nabla \Upsilon=-\nabla \Phi+\mathbf{b} ; \quad \nabla \cdot \mathbf{b}=0
$$

where

$$
\Phi=e_{0} / r
$$

is the usual Coulomb potential of the charge $e_{0}$ in spherical polar coordinates $\{r, \vartheta, \varphi\}$. A particular solution is obtained when b equals some constant vector $\mathfrak{E}$. Since $\psi$ should asymptote to a constant at infinity, $\mathfrak{E}$ is obviously the applied external electric field up to a positive proportionality constant. The curl of Eq. (19) gives $\nabla \psi \times \nabla \Upsilon=0$ which shows that $\psi$ and $\Upsilon$ are functions of each other. By Eq. (19) both can be taken as functions of only the potential $V \equiv \Phi-\mathfrak{E} \cdot \mathbf{x}$. Thus

$$
\mathbf{E}=-e^{2 \psi} \nabla V
$$

with $\psi=\psi(V)$. So far it is clear that Eq. (21) is the unique solution when $\mathbf{b}=\mathfrak{E}$. But as argued in Sec. IV A, the uniqueness survives when $\mathbf{b}$ is only known to asymptote to $\mathfrak{E}$.

In view of Eq. (21),

$$
\nabla^{2} \psi=\psi^{\prime} \nabla^{2} V+\psi^{\prime \prime} e^{-4 \psi} \mathbf{E}^{2}
$$

( $\equiv d / d V$, etc). Since $\nabla^{2} V=-4 \pi e_{0} \delta^{3}(\mathbf{x})$, the first (second) term on the r.h.s. of Eq. (22) must match the first (second) term on the r.h.s. of Eq. (18). The second condition, namely,

$$
\psi^{\prime \prime}=\kappa^{2} e^{2 \psi}
$$

can be integrated after multiplication by $\psi^{\prime}$ to give

$$
\psi^{\prime 2}=\kappa^{2}\left(e^{2 \psi}+\varpi\right)
$$

with $\varpi$ a dimensionless constant of integration. Appendix A obtains all solutions of this equation.

Identifying the pointlike source terms of Eqs. (18) and (22) gives

$$
\kappa^{2}\left(\partial m c^{2} / \partial \psi\right)=-e_{0} \psi^{\prime} \quad \text { at } \quad \mathbf{x}=0 .
$$

This condition makes the two source terms equivalent even when the $\delta$ function is somewhat smeared (I shall show below that in this theory there is a lower bound to the radius of any charge, so this smearing must occur). As evident from the appearance in the r.h.s. of a term linear in $e_{0}$, the dependence arising from this equation is not automatically of the form $m=m_{0}+$ const. $\times e_{0}^{2} e^{2 \psi}$ commonly adopted (Coulomb model).

\section{B. Physical choice of $\varpi$ and WEP}

To find out about $\varpi$ I now work out the total force of electric origin acting on the charge $e_{0}$. I make no attempt to separate out the self field. Immediately after the charge is released Eqs. (13) and (21) give

$$
\begin{aligned}
m d \mathbf{v} / d t & =-\left(\partial m c^{2} / \partial \psi\right) \boldsymbol{\nabla} \psi+e_{0} \mathbf{E} \\
& =-\left[\left(\partial m c^{2} / \partial \psi\right) \psi^{\prime}+e_{0} e^{2 \psi}\right] \boldsymbol{\nabla} V .
\end{aligned}
$$

It is to be stressed that the force on the r.h.s. here comprises both the (modified) Coulomb force and the anomalous force. Substituting $\left(\partial m c^{2} / \partial \psi\right)$ from Eq. (25) and $\psi^{\prime 2}$ from Eq. (24) gives, after a cancellation, that

$$
m d \mathbf{v} / d t=\varpi e_{0} \nabla V
$$

The choice $\varpi=0$ in the solution corresponding to a charge $e_{0}$ is physically untenable. It would mean that in the presence of an arbitrary external field $\mathfrak{E}, e_{0}$ experiences no force whatsoever. This is contrary to all experience, and the theory developed here is supposed to describe the real world. It must be, then, that $\varpi \neq 0$. For $\varpi \neq 0$ the force on the r.h.s. includes the usual self-force term proportional to $\nabla \Phi$ which is always dropped in Maxwellian electrodynamics; I do so too. Thus

$$
m d \mathbf{v} / d t=-\varpi e_{0} \mathfrak{E}
$$


It would be hasty to reject outright all the cases with $\varpi \neq-1$ on the grounds that Eq. (28) would then have the wrong form; after all $\mathfrak{E}$ is the external electric field only up to a positive proportionality constant. However, with $\varpi>0$ the net force acting on the particle would be opposite the accepted one. One cannot remove this problem by assuming that the "passive" charge here which senses the applied field is opposite in sign to the "active" charge $e_{0}$ which is the source of the electric field in Eq. (17). For if this were true here, it would be true in other cases too. If $\mathfrak{E}$ is then interpreted as representing the field of a distant charge (up to a positive multiplier), it is immediately apparent that charges of one sign would attract. I thus conclude that physically $\varpi$ must be negative.

In Appendix A all solutions of Eq. (24) are found; that for negative $\varpi$ is ( $\chi$ is an integration constant):

$$
e^{\psi}=|\varpi|^{1 / 2} \sec \left(|\varpi|^{1 / 2} \kappa V+\chi\right)
$$

This solution is unique (in the physical sense) by the one-to-one correspondence between solutions of Eq. (24) for one charge and the solutions of the corresponding equation for many charges (see Sec. IV A). The latter are certified as a complete set of solutions by the duality argument to be set forth in Sec. VI A, and the particular solution with $\varpi<0$ corresponds to Eq. (29).

Let us interpret $e_{p} \equiv|\varpi|^{1 / 2} e_{0}$ as the physical charge and $\mathfrak{E}_{p} \equiv|\varpi|^{1 / 2} \mathfrak{E}$ as the physical external field. This brings Eq. (28) to precisely the everyday form of the Newtonian equation of motion in an external field; $\varpi$ disappears from the equation of motion.

Eq. (29) now takes the form

$$
e^{\psi}=|\varpi|^{1 / 2} \sec \left(\kappa V_{p}+\chi\right)
$$

where $V_{p} \equiv \Phi_{p}-\mathfrak{E}_{p} \cdot \mathbf{x}$, and $\Phi_{p}$ is built just as $\Phi$ in Eq. (20) but from the physical charge. It would seem that $\varpi$ still appears after the reinterpretation. However, this is just an illusion. Consider the energy density of the electric field, $\mathcal{E}=(8 \pi)^{-1} e^{-2 \psi} \mathbf{E}^{2}$ according to Eq. (B2). This can be recast in terms of physical quantities in such a way that $\varpi$ does not appear explicitly:

$$
\mathcal{E}=(8 \pi)^{-1} \sec ^{2}\left(\kappa V_{p}+\chi\right) \cdot\left(\mathfrak{E}_{p}-\nabla \Phi_{p}\right)^{2}
$$

The same can be said about the purely Coulomb force on the test charge, $\mathfrak{F}=e_{0} \mathbf{E}$, which takes the form

$$
\mathfrak{F}=e_{p} \sec ^{2}\left(\kappa V_{p}+\chi\right) \cdot\left(\mathfrak{E}_{p}-\nabla \Phi_{p}\right)
$$

Again $\varpi$ has disappeared. All this means that setting $\varpi=-1$ (identifying $e_{0}$ with the physical charge) is a matter of convention, and does not entail any assumptions about the physics. I thus take $\varpi=-1$ and drop the subscript $p$ henceforth.

The equation of motion (28) for the particle in an external field (with $\varpi=-1$ ) already shows that the WEP is not violated - at least not in an obvious way — in the theory in question. The putative anomalous force from the $\psi$ dependence of the mass is compensated for by the modification of the Coulomb force effected by the $\psi$ field [the $e^{2 \psi}$ factor in Eq. (26)] in such a way that, at least for a quasistationary charged particle, the force acting on the charge is independent of the fraction of its rest mass which is of Coulomb provenance. One finds no indication here for a violation of the WEP.

A brief remark about neutral particles is in order here. In that case Eqs. (17)-(18) reduce to a Poisson equation for $\psi$ with a pointlike source. The formal solution is $\psi=C / r$ with $C$ a constant proportional to $\partial m / \partial \psi$ of the source. Dvali and Zaldarriaga $[12,25]$ suggest that $C$ is generically nonzero with the consequence that long-range anomalous forces exist even between neutral particles. For the neutron they infer this from the $\alpha$ dependence of the neutron mass coming from virtual photon exchange between its constituent quarks [12] as calculated perturbatively to low order. As discussed in Sec. IV A, the framework suggests rather that $C=0$, at least when some charged particles are also present.

\section{Minimal size of an isolated charge}

Henceforth I set $\mathfrak{E}=0$. With $\varpi=-1$, Eq. (30) gives

$$
e^{\psi}=\sec (\kappa \Phi+\chi)
$$

Apart from the inclusion of the "phase" $\chi$, this is the original solution presented in Ref. [9], where it was pretty much gotten by guessing. Redefining $\chi$ is equivalent to shifting the zero of $\Phi$; this is certainly without physical significance here as in Maxwellian electrodynamics. As already clear from Eq. (20), I here adhere to the convention that $\Phi$ 
vanishes at infinity. The value of $\chi$ is thus fixed by the asymptotic value of $e^{\psi}$, which coincides with the instantaneous cosmological value of $e^{\psi}$ in the appropriate model of the universe.

According to Sec. II, it is permissible to multiply all $e_{0 i}$ by a common positive constant while simultaneously dividing $\epsilon$ by it. I exploit this freedom to set the cosmological value of $\epsilon$ to unity at the present epoch. After this is done one can define $\psi$ as the logarithm of $\epsilon$ and pass to the second form of the theory. From the solution (33) at the mentioned epoch one then finds that $\chi=0$ (this was also the choice of Ref. [9]). Although $\chi$ evolves cosmologically, it is evidently possible to set $\chi=0$ at any one cosmological epoch. Thus results we shall obtain below that depend on having $\chi=0$ are valid at any single epoch. It is only when interests centers on comparing physics at two separate epochs that one can no longer do away with $\chi$.

By Eq. (33) $e^{\psi}$ can diverge and then turn negative when $|\Phi| \geq \pi^{3 / 2}(\hbar c)^{1 / 2} l^{-1}$. Because the permittivity of the vacuum cannot be negative, this must mean that $|\Phi|$ can never reach such values, i.e., that the particle with charge $e_{0}$ is spread over a sufficiently large radius $R$ to prevent this. By Eq. (20) this condition is

$$
R>\pi^{-3 / 2}\left(e_{0}^{2} / \hbar c\right)^{1 / 2}\left(l / \ell_{P}\right) \ell_{P} .
$$

Because asymptotically $e^{\psi} \rightarrow 1$ at the present epoch, one can take $\left(e_{0}^{2} / \hbar c\right)^{1 / 2}=(137)^{-1 / 2} \sim 0.1$ for $e_{0}$ the elementary charge. And by the framework's assumption $(7), l>\ell_{P}$; in fact I shall show in Sec. VII that if the alleged cosmological $\alpha$ variability is to find explanation in this framework, $l$ must be an order of magnitude above $\ell_{P}$ (see also Refs. $[9,13,14,15])$. Thus the lower bound on the radius of any charge is at least a Planck length. Composite particles, e.g. the proton, easily satisfy Eq. (34). For leptons and quarks which are regarded as pointlike, quantum gravitational effects must intervene at radii of a few Planck lengths and modify the above classical considerations. But it is noteworthy that our formal lower bound on $R$ is not at variance with the widespread belief that no elementary particle can be smaller than $\ell_{P}$, the scale at which spacetime can no longer be regarded as a continuum.

\section{Why neglect spacetime curvature ?}

The neglect of spacetime curvature in all the preceding calculations may be justified when the particle in question is either elementary or made up of a not excessive number of elementary particles. Appendix B shows that corrections to the usual exterior Reissner-Nordström metric (of general relativity) belonging to a particle with charge $e_{0}$ become important in our theory only when $|\Phi|$ is no longer small compared to $\pi^{3 / 2}(\hbar c)^{1 / 2} l^{-1}$. By Eq. $(34)|\Phi|$ gets that big only as $r$ approaches $R$. Thus, if all one wants is to investigate the source's exterior, one can, with good accuracy, employ the Reissner-Nordström metric,

$$
\begin{aligned}
d s^{2} & =-e^{-\lambda} d t^{2}+e^{\lambda} d r^{2}+r^{2}\left(d \theta^{2}+\sin ^{2} \theta d \varphi^{2}\right) \\
e^{-\lambda} & \equiv 1-2 G m c^{2} r^{-1}+G e_{0}^{2} c^{-4} r^{-2}
\end{aligned}
$$

where $m$ is the source's mass.

This metric begins to departs seriously (5\% level) from Minkowski's at a radius $r \sim \max \left(5 G^{1 / 2} e_{0} c^{-2}, 40 G m c^{-2}\right)$. Now for the known charged elementary particles or small agglomerates of them, $m \ll G^{-1 / 2} e_{0}$ (in fact $G^{-1 / 2} e_{0} / m$ is of order $10^{25}$ for the electron and $10^{20}$ for a nucleus or atom). Thus significant departures from flatness are only found at $r<5 G^{1 / 2} e_{0} c^{-2}=5\left(e_{0}^{2} / \hbar c\right)^{1 / 2} \ell_{P}$, i.e., at Planck scale where the whole classical description is already irrelevant. And as pointed out in Sec. III C, pointlike particles in this framework cannot be smaller than this. Thus, description of the exterior of a pointlike elementary object (or a small collection of such) can well afford to ignore spacetime curvature.

\section{MULTIPLE ELECTRIC CHARGES}

\section{A. The solution}

The results above may be generalized to a collection of $N$ charges $e_{0 i}$ initially clamped at positions $\mathbf{z}_{i}, i=1,2, \cdots, N$. Again with neglect of gravity Eqs. (14-(16) reduce to

$$
\begin{array}{r}
\boldsymbol{\nabla} \cdot\left(e^{-2 \psi} \mathbf{E}\right)=4 \pi \sum_{i} e_{0 i} \delta^{3}\left(\mathbf{x}-\mathbf{z}_{i}\right) \\
\nabla^{2} \psi=4 \pi \kappa^{2}\left[\sum_{i} \frac{\partial m_{i} c^{2}}{\partial \psi} \delta^{3}\left(\mathbf{x}-\mathbf{z}_{i}\right)+\frac{1}{4 \pi} e^{-2 \psi} \mathbf{E}^{2}\right]
\end{array}
$$


As in Sec. III, I take $\mathbf{E}=\nabla \Upsilon$.

I define anew

$$
\Phi(\mathbf{x})=\sum_{i} \frac{e_{0 i}}{\left|\mathbf{x}-\mathbf{z}_{i}\right|}
$$

this is the standard Coulomb potential due to all the charges. Recalling that

$$
\nabla^{2} \Phi=-4 \pi \sum_{i} e_{0 i} \delta^{3}\left(\mathbf{x}-\mathbf{z}_{i}\right)
$$

it is now easy to check that

$$
\mathbf{E}=-e^{2 \psi} \nabla \Phi
$$

together with any of the choices ( $\chi$ is a constant)

$$
e^{\psi}=\left\{\begin{array}{cl} 
\pm(\kappa \Phi+\chi)^{-1} ; & \varpi=0 \\
\pm \sqrt{ } \varpi \operatorname{csch}(\sqrt{ } \varpi \kappa \Phi+\chi) ; & \varpi>0 \\
\sqrt{ }|\varpi| \sec (\sqrt{ }|\varpi| \kappa \Phi+\chi) ; & \varpi<0
\end{array}\right.
$$

constitute solutions of Eqs. (37)-(38) provided the analog of Eq. (25) is satisfied for every one of the charges, to wit

$$
\kappa^{2}\left(\partial m_{i} c^{2} / \partial \psi\right)=-e_{0 i} \psi^{\prime} \quad \text { at } \quad \mathbf{x}=\mathbf{z}_{i} .
$$

I shall exploit this last equation in Appendix $\mathrm{C}$ to elucidate the spatial variation of the $m_{i}$.

It turns out that Eqs. (41) and (42) comprise all the static solutions for multiple charges. Although it is hard to prove this directly, results in Sec. VI A enable this be established immediately by duality arguments. The one-to-one correspondence between the branches of $\psi$ in Eq. (42) and those for the one-particle solution (A5) then establishes that in Sec. III B, Eq. (21) is indeed the most general form for $\mathbf{E}$ possible even if $\mathbf{b}$ is not assumed constant except asymptotically; generalizations of Eq. (21) would necessarily affect $\psi$ thorough Eq. (18).

Since the physical multiparticle solution should include the physical single particle solution as a special case, I put $\varpi=-1$ here as in Sec. IIIB. I also set $\chi=0$ on the basis of the argument of Sec. IIIC. That this choice of parameters is consistent is made clear by the following argument. Suppose $\Phi_{i>1}$, the part of $\Phi$ coming from particles $i=2,3, \cdots, N$, is approximated by the first two terms of a Taylor series about $\mathbf{z}_{1}$. Denoting $\kappa \Phi_{i>1}\left(\mathbf{z}_{1}\right)$ by $\chi_{1}$ and $\nabla \Phi_{i>1}$ at $\mathbf{z}_{1}$ by $-\mathfrak{E}$, the last of Eqs. (42) gives in the vicinity of charge $i=1$

$$
e^{\psi} \approx \sec \left\{\kappa\left[\Phi_{i=1}-\mathfrak{E} \cdot\left(\mathbf{x}-\mathbf{z}_{1}\right)\right]+\chi_{1}\right\} .
$$

This is of the same form as Eq. (30) for the single charge solution. Although the phase $\chi$ of the whole charge complex has been set to zero, each individual charge has an associated phase, $\chi_{i}$, induced by its neighbors. The result (44) makes it clear again that the one-charge solution Eq. (30) is physically unique, as mentioned earlier.

With the above choices the full multiparticle solution is

$$
\begin{aligned}
e^{\psi} & =\sec (\kappa \Phi) \\
\mathbf{E} & =-e^{2 \psi} \nabla \Phi=-\kappa^{-1} \nabla \tan (\kappa \Phi)
\end{aligned}
$$

This result serves to clear up the question (Sec. IIIB) about the value of $\partial m / \partial \psi$ for a neutral particle. Because neutrinos and neutral mesons are scarce in laboratory matter, the particle of most interest is the neutron. It is composite, but I avoid any discussion of its structure and extension, and treat it as pointlike object as it would indeed appear to low energy probes. In this spirit one can include a neutron as particle $n$ in the collection just discussed by formally taking $e_{0 n} \rightarrow 0$. It is easy to verify that this limit is a solution of the equations. It follows from Eqs. (45)-(46) that $\psi$ is regular at the neutral's position, and from Eq. (43) that $\partial m_{n} / \partial \psi=0$. More generally, for a neutral pointlike particle, $m$ is $\psi$ (and spacetime) independent. It remains a task for the future to reconcile this conclusion with the dependence of the neutron mass on $\alpha$ according to perturbative calculations within QCD [12].

\section{B. The momentum equation and WEP}

In our context the WEP would require that a composite possibly charged body moves in uniform external electric and gravitational fields with an acceleration which depends only on its total mass and charge, but not on its detailed 
inner structure. To put the framework to the test in this respect, I imagine all $N$ charges in "the world" to be lumped into two clusters. One, a spherical massive one, I put at the origin and regard it as a single particle of mass $m_{1}$ and charge $e_{01}$. The other, denoted by $\mathcal{C}$, is made up of $N-1$ charges $e_{0 i}$ with masses $m_{0 i} ; i=2,3, \ldots N$. By assuming the distance between the clusters is large compared to both their extensions, it is possible to think of the charge at the origin as pointlike, and $\mathcal{C}$ as immersed in the uniform fields of the former.

I take the static solution for the fields, Eqs. (45)-(46), as part of the initial conditions for the envisaged dynamical situation. I restrict discussion to the initial moment $t=0$ when all the charges are still at rest. This restriction is necessary here because the fully dynamical solution is as yet unknown. However, it should be clear that any violations of the WEP would be expected to show up already at the initial moment because they involve the acceleration. The demonstration in Sec. IV C that no such violations occur, at least within the approximation to be described presently, strongly suggests that the WEP holds to great accuracy in the dynamical situation as well.

As a first step I calculate the rate of change of total momentum in the masses constituting $\mathcal{C}$. According to Eq. (12) in the nonrelativistic approximation and with neglect of gravity

$$
\frac{d}{d t} \sum_{i=2}^{N} m_{i} \mathbf{v}_{i}=\sum_{i=2}^{N}\left(-\frac{\partial m_{i} c^{2}}{\partial \psi} \nabla \psi+e_{0 i} \mathbf{E}\right)_{\mathbf{x}=\mathbf{z}_{i}} .
$$

Substitution from Eqs. (43) and (45)-(46) and use of the identity $\sec ^{2} x-\tan ^{2} x=1$ transforms this into

$$
\frac{d}{d t} \sum_{i=2}^{N} m_{i} \mathbf{v}_{i}=-\left.\sum_{i=2}^{N} e_{0 i} \nabla \Phi\right|_{\mathbf{x}=\mathbf{z}_{i}}
$$

Now near charge $i, \nabla \Phi$ is dominated by the self-field $\left(\mathbf{x}-\mathbf{z}_{i}\right) /\left|\mathbf{x}-\mathbf{z}_{i}\right|^{3}$ which points radially out from it. As in Maxwellian electrodynamics, here one may think of the force from this part as averaging out in the limit. Left over from the force are the terms $(j \neq i)$

$$
\sum_{i=2}^{N} \sum_{j=1}^{N} \frac{e_{0 i} e_{0 j}\left(\mathbf{z}_{i}-\mathbf{z}_{j}\right)}{\left|\mathbf{z}_{i}-\mathbf{z}_{j}\right|^{3}}=e_{01} \sum_{i=2}^{N} \frac{e_{0 i}\left(\mathbf{z}_{i}-\mathbf{z}_{1}\right)}{\left|\mathbf{z}_{i}-\mathbf{z}_{1}\right|^{3}}
$$

with the second form following cancellation of the $j \neq 1$ terms in pairs. The assumed smallness of $\mathcal{C}$ as compared with the distances $\left|\mathbf{z}_{i}-\mathbf{z}_{1}\right|$ justifies replacement of every $\mathbf{z}_{i}$ on the r.h.s. by the cluster's center-of-mass position $\mathbf{Z}$ (an approximation no different from the one customarily made in Maxwellian systems):

$$
\frac{d}{d t} \sum_{i=2}^{N} m_{i} \mathbf{v}_{i}=Q \frac{e_{01}\left(\mathbf{Z}-\mathbf{z}_{1}\right)}{\left|\mathbf{Z}-\mathbf{z}_{1}\right|^{3}}
$$

with $Q \equiv \sum_{i=2}^{N} e_{0 i}$. Thus the rate of change of momentum of the particles in the cluster is controlled by the formal Coulomb field of charge $i=1$, approximated as uniform at $\mathcal{C}$, and by $\mathcal{C}$ 's total charge. Just as in Maxwellian electrodynamics, here the cluster's internal structure does not affect the rate of change of its total particle momentum.

Although this finding is consistent with the claim that the WEP is satisfied here, it is no complete proof: the $m_{i}$ vary, making the relation between rate of change of the total particle momentum and the acceleration of the center-of-mass' less clear than usual.

\section{Restricted microscopic proof of WEP}

To clarify the above point I look at the time component of energy-momentum conservation for the whole system $(\mathcal{C}$ and charge $i=1$ ). If $T^{\mu \nu}$ includes the tensors for the particles and the electromagnetic and scalar fields, $T_{; \nu}^{\mu \nu}=0$ can be derived from the gravitational field equations as usual. Ignoring the gravitational field gives

$$
\partial T^{00} / \partial t+\partial T^{0 i} / \partial x^{i}=0 .
$$

Multiplying the equation by $x^{j}$, integrating over a large volume $\mathcal{V}$ containing $\mathcal{C}$ but excluding charge 1 , and integrating by parts gives

$$
\frac{d}{d t} \int_{\mathcal{V}} T^{00} x^{j} d^{3} x=\int_{\mathcal{V}} T^{0 j} d^{3} x-\oint_{\partial \mathcal{V}} x^{j} T^{0 i} d^{2} S_{i}
$$


The integral on the l.h.s. is just the product of total mass $M=\int_{\mathcal{V}} T^{00} d^{3} x$ of the cluster (neglecting contributions from the fields beyond the reaches of $\mathcal{C}$ ) and the component $Z^{j}$ of $\mathbf{Z}$. One further time derivative gives

$$
\frac{d^{2}}{d t^{2}} \int_{\mathcal{V}} T^{00} x^{j} d^{3} x=\frac{d}{d t} \int_{\mathcal{V}} T^{0 j} d^{3} x-\frac{d}{d t} \oint_{\partial \mathcal{V}} x^{j} T^{0 i} d^{2} S_{i}
$$

The spatio-temporal component $T^{0 i}$ figuring in Eq. (53) receives three contributions. The first, $T_{(f)}{ }^{0 i}$ from the $f^{\mu \nu}$ field (see Appendix B), comprises products of $f^{0 i}$ with $f^{i j}$. The $f^{i j}$ evidently vanish at $t=0$ (no motion, no magnetic field). Further, because I assume the static solution (45)-(46) for $\psi$ and $\mathbf{E}$ holds at $t=0, f_{0 i}=-c E^{i}$ is a gradient. Then the identity $\dot{f}_{i j}+f_{0 i, j}+f_{j 0, i}=0\left(f_{\mu \nu}\right.$ derives from a 4-potential) shows that initially $\dot{f}_{i j}=0$. Therefore, time differentiation anihilates both integrals over $T_{(f)}{ }^{0 i}$ in Eq. (53) at $t=0$ (that $\dot{\psi}=0$ at $t=0$ will be shown presently).

The second contribution, $T_{(\psi)}{ }^{0 i}$ (again see Appendix B), comprises products of $\psi^{, i}$ with $\dot{\psi}$. Now because the scalar equation (16) is of second order in time, one may require $\dot{\psi}=0$ at $t=0$; this is consistent with this instant representing the end of a purely static situation. Further, comparison of Eqs. (38) and (16), the latter satisfied identically by solution (45)-(46), shows that also $\ddot{\psi}=0$ at $t=0$ for the assumed initial conditions. Thus time differentiation of both integrals over $T_{(\psi)}{ }^{0 i}$ in Eq. (53) fails to produce nonvanishing contributions at $t=0$.

Accordingly, the time derivatives at $t=0$ in Eq. (53) of both integrals over $T^{0 i}$ come solely from the particles in $\mathcal{C}$. Since the surface $\partial \mathcal{V}$ lies beyond them, the r.h.s. of Eq. (53) comprises solely $\frac{d}{d t} \int T_{\mathrm{m}}{ }^{0 j} d^{3} x$. The tensor in question is derived from action (11) by variation of $g^{\mu \nu}$ and use of $g_{\mu \nu} u^{\mu} u^{\nu}=-c^{2}$ :

$$
T_{\mathrm{m}}{ }^{\mu \nu}=\sum_{i} m_{i} c \frac{d z_{i}^{\mu}}{d \tau} \frac{d z_{i}^{\nu}}{d \tau} \frac{\delta^{3}\left(\mathbf{x}-\mathbf{z}_{i}(\tau)\right)}{\gamma \sqrt{ }-g}
$$

Neglecting the difference between $\tau$ and $t$, replacing $\sqrt{ }-g$ by its Minkowski value $c$, and substituting in Eq. (53) gives

$$
\frac{d^{2}\left(M Z^{j}\right)}{d t^{2}}=\frac{d}{d t} \sum_{i=2}^{N} m_{i} \mathbf{v}_{i} .
$$

I complete the derivation by showing that at $t=0 \mathrm{M}$ can be taken out from under the derivatives. Integrating Eq. (51) over $\mathcal{V}$ and using Gauss' theorem gives

$$
\frac{d}{d t} \int_{\mathcal{V}} T^{00} d^{3} x=-\oint_{\partial \mathcal{V}} T^{0 i} d^{2} S_{i}
$$

Differentiating this result by $t$ gives

$$
\frac{d^{2}}{d t^{2}} \int_{\mathcal{V}} T^{00} d^{3} x=-\frac{d}{d t} \oint_{\partial \mathcal{V}} T^{0 i} d^{2} S_{i}
$$

By precisely the same arguments given above, the r.h.s. of these two equations vanish at $t=0$. Since the integral in both l.h.s. is $\mathcal{C}$ 's total mass $M$, this demonstrates that $\dot{M}=\ddot{M}=0$ at $t=0$. Of course this does not mean that $M$ is a conserved quantity, only that it behaves as $M(t=0)+\mathcal{O}\left(t^{3}\right)$ for short times. Substituting this result in Eq. (55) and taking Eq. (50) into account gives at $t=0$

$$
\ddot{\mathbf{Z}}=\frac{Q}{M} \frac{e_{01}\left(\mathbf{Z}-\mathbf{z}_{1}\right)}{\left|\mathbf{Z}-\mathbf{z}_{1}\right|^{3}} .
$$

Thus in harmony with the WEP, the acceleration of $\mathcal{C}$ 's center-of-mass in the field of the distant charge $e_{01}$ is fully determined by its mass $M$ and total charge $Q$, and is insensitive to its structure (disposition of the member charges, their charge to mass ratios, etc.) To reach this result the mass $M$ of $\mathcal{C}$ had to be identified with the integral of $T^{00}$ taken over a finite, albeit large, region. This is a necessity in any situation when the system of interest is not the only one in the universe; the same procedure would be required in any other field theory. Another limitation of the approach is that result (58) is rigorously valid only when all particles in $\mathcal{C}$ are assumed to be at rest (thus the approach neglects purely magnetic effects). But intuitively the acceleration's universality property should remain valid if all velocities are small and spin magnetism is weak, as I indeed show in Secs. V B and VIB.

The results here concur with those reached by Landau, Sisterna and Vucetich [19] on the basis of the TH $\epsilon \mu$ formalism, a nonrelativistic generic parametrized microscopic description of the gravitational, particle and electromagnetic sectors of field theories. The authors find a connection between the lack of overall charge conservation and violations of the 
WEP. Looking at the present framework in the light of experimental constraints on charge nonconservation, they are able to certify that WEP is respected to a fractional accuracy (in the acceleration) $\sim 10^{-18}$, beyond the projected sensitivity of WEP tests in the foreseeable future. Since charge conservation is actually exact in the framework [see the comments after Eq. (15)] the implication is actually stronger: no WEP violations are expected, at least nonrelativistically, even when slow motion of charges is allowed.

There exist other possibilities for variability of $\alpha$ by way of a scalar field which do not run counter to the EDB experiments. Such is the supersymmetric grand unified theory of Chacko, Grojean and Perelstein [26], according to which a late epoch cosmological phase transition causes a jump in $\alpha$ while generating a vacuum expectation value of the scalar field which makes it short ranged. Consequently, although a charged particle's mass can be scalar field dependent, this does not lead to long range anomalous forces which would contradict the said experiments.

\section{MACROSCOPIC DESCRIPTION OF ELECTRIC STRUCTURE AND WEP}

A virtue of the "proof" of the WEP in Sec IV C is that it does not require knowledge of the equations of motion for macroscopic matter [as opposed to the microscopic level Eq. (13)]. Its shortcomings are that it establishes the validity of the WEP only for brief time intervals after a putative quiescent situation, and only when the magnetic structure of the systems involved is ignored. Both shortcomings will now be remedied. By averaging over microscopic quantities to produce a macroscopic (or continuum) version of the theory, I show in Sec. V B that the WEP-breaking effects pointed out in Refs. $[6,7,9,10,11,12,13]$ are ruled out if one is willing to assume that a macroscopic chunk of matter moves according to Eq. (13) with a natural definition of $m(\psi)$ obtained by macroscopic averaging. No short-times assumption is then necessary. And in Sec. VIB magnetic dipole structure of matter is incorporated into the arguments.

Secs. III andIV describe matter microscopically, that is as a collection of pointlike particles $i$ with definite charges $e_{0 i}$ (some of which may be zero) and masses $m_{i}$ subject to the relation (43). One key assumption I make on the way to the macroscopic description is that the many charges solution (45)-(46) remains valid, at least approximately, when the particles move slowly. I define the spatial average $\overline{\mathcal{Q}}$ of a quantity $\mathcal{Q}$ as the integral of $\mathcal{Q}$ over some macroscopic region of volume $\mathcal{V}$, all divided by $\mathcal{V}$. In flat spacetime or in local Lorentz frames (assumed to be large enough to encompass the said macroscopic region), no ambiguity in this definition arises from issues of parallel transport of vectors and the like.

Another assumption I make is that for a macroscopic test body $\mathcal{T}$ of mass $\mu$ and charge $q$ moving on background scalar, Newtonian and electric fields, $\bar{\psi}, \boldsymbol{\nabla} \phi_{N}$ and $\mathfrak{E}$ (all regarded as approximately uniform), moves according to Eq. (13) [Eq. (26) in the nonrelativistic case]:

$$
\mu d \mathbf{v} / d t=-\mu \boldsymbol{\nabla} \phi_{N}-\left(\partial \mu c^{2} / \partial \bar{\psi}\right) \boldsymbol{\nabla} \bar{\psi}+q \mathfrak{E}
$$

The assumption would be a triviality but for the stipulation that $\mu(\bar{\psi})$ is to be identified with the macroscopic average of the total energy density in $\mathcal{T}$ multiplied by its volume. Evidently $q$ is to be interpreted as the sum of charges in $\mathcal{T}$, while $\mathfrak{E}$ is the electric field determined from its sources by Eq. (37) and averaged over the volume of $\mathcal{T}$. The only quantity which requires special discussion is $\boldsymbol{\nabla} \bar{\psi}$.

\section{A. The Coulomb model for $\bar{\psi}$}

In the language of the present framework, Dicke's argument [6] amounts to assuming that the macroscopic field $\bar{\psi}$ is related to its source $\mathcal{S}$ via a macroscopic version of Eq. (38) with the sum of $\left(\partial m_{i} c^{2} / \partial \psi\right) \delta^{3}\left(\mathbf{x}-\mathbf{z}_{i}\right)$ replaced by $2 \zeta \rho c^{2}$, where $\zeta$ is the typical fraction of the source's mass density $\rho$ which is of Coulomb provenance. This comes from assuming that $\rho \propto \alpha \propto e^{2 \bar{\psi}}$. Now the second term in the square brackets in Eq. (38) is, according to Appendix B, exactly twice the density of electric energy, just as the former contribution. Hence in the Coulomb model for the source, Eq. (38) takes the form

$$
\nabla^{2} \bar{\psi}=16 \pi \kappa^{2} \zeta \rho c^{2}=\left(l / \ell_{P}\right)^{2}\left(\zeta / \pi c^{2}\right) 4 \pi G \rho,
$$

A likeness of this equation and/or its cosmological version reappears in subsequent treatments $[9,10,11,13,14,15$, $16,17,18]$.

The similarity of Eq. (60) with Poisson's equation for the Newtonian potential of $\mathcal{S}$, and the similarity between the asymptotic boundary conditions on $\phi_{N}$ and $\bar{\psi}$, permits the identification

$$
\boldsymbol{\nabla} \bar{\psi}=\left(l / \ell_{P}\right)^{2}\left(\zeta / \pi c^{2}\right) \nabla \phi_{N}
$$


Actually, in a nonspherical configuration the curl of some vector may be added to the r.h.s.; however, this "correction" must decay asymptotically as $1 / r^{3}$ (cf. Ref. 27, Appendix A) and so should become irrelevant some distance outside $\mathcal{S}$. Eq. (61) shows that $\nabla \alpha / \alpha=\left(l / \ell_{P}\right)^{2}\left(2 \zeta / \pi c^{2}\right) \nabla \phi_{N}$. With $l \sim \ell_{P}$ this result is very much like Dicke's conjectured one [6] mentioned in Sec. I, except for the replacement of $\alpha$ by $2 \zeta$, which for ordinary matter is of the same order as $\alpha$.

How does this all bear on the WEP ? Suppose $\mathcal{T}$ moves in the vicinity of $\mathcal{S}$. In the Coulomb model one replaces $\left(\partial \mu c^{2} / \partial \psi\right)$ in the equation of motion for $\mathcal{T}$, Eq. (59), by $2 \zeta^{\prime} \mu c^{2}$, where $\zeta^{\prime}$ is now the Coulomb energy fraction of $\mu c^{2}$. With the replacement (61) this gives (in the absence of other sources)

$$
d \mathbf{v} / d t=-\left[1+\left(l / \ell_{P}\right)^{2}\left(2 \zeta \zeta^{\prime} / \pi\right)\right] \nabla \phi_{N}+q \mathfrak{E},
$$

a version of which first appeared in Ref. [9]. Now, as mentioned in Sec. I, for ordinary matter $\zeta$ is of order a few times $10^{-3}$ and varies by about $10^{-3}$ from material to material. The latest EDB tests of WEP find that $d \mathbf{v} / d t$ is $\zeta^{\prime}$ independent to fractional accuracy $10^{-13}$ [8]. This is consistent with Eq. (62) only if $l$ is $10^{-3} \ell_{P}$ or smaller. Because of this and assumption (7) of the framework, I inclined in Ref. [9] to the opinion that there is no $\alpha$ variability in nature (as would be the case if $l \equiv 0$, e.g. electrodynamics exactly Maxwellian).

This was also the conclusion of Livio and Stiavelli [10] who noted the difficulty in explaining the alleged cosmological $\alpha$ variability with $l$ as small as $10^{-3} \ell_{P}$ and the accepted matter content of the universe. Olive and Pospelov [11] also took cognizance of this problem; to solve it they proposed that the cosmological dark matter is much more strongly coupled to the $\psi$ field than is ordinary matter. This would have the effect of counteracting the smallness of $l / \ell_{P}$ inferred from tests of the WEP. By contrast, Magueijo, Barrow and Sandvik [13] see no immediate strong contradiction between the EDB experiments tests; they infer $\left(l / \ell_{P}\right)^{2} \approx 10^{-4}$ from the claimed cosmological $\alpha$ variability by assuming that cosmological matter has $\zeta \approx 1$. They further adopt, for ordinary matter, the very low values $\zeta \sim \zeta^{\prime} \sim 10^{-4}$. However, these run on the face of simple estimates from nuclear Coulomb energy (see Sec. V B 2). There is thus a tension between the claimed cosmological $\alpha$ variability and the tests of the WEP. In the next subsections I show that the blame for the above impasse lies squarely with the misuse of the Coulomb model, there being no need to take $l<\ell_{P}$. When needed in what follows I assume $l$ is above $\ell_{P}$ and within an order of magnitude of it.

\section{B. Coulomb energy and WEP violation}

For a quick orientation one can describe the detailed electric structure of the source $\mathcal{S}$ of $\bar{\psi}$ with the static electric solution, Eqs. (39), (45-(46). One is thus neglecting effects of internal motions and magnetic structure, both usually minor complications for nonrelativistic sources of interest.

I assume that $\kappa|\Phi| \ll 1$ everywhere and check this on a case-by-case basis. Then Taylor expanding both sides of Eq. (45) and averaging as stipulated in the preamble of this section gives

$$
\bar{\psi} \approx \frac{1}{2} \kappa^{2} \overline{\Phi^{2}}=\frac{1}{2} \kappa^{2} \bar{\Phi}^{2}+\frac{1}{2} \kappa^{2} \overline{(\Phi-\bar{\Phi})^{2}}
$$

Note that matter with electric structure causes the physical $\alpha$, which is proportional to $e^{2 \psi} \approx 1+2 \psi$, to be slightly larger nearby than asymptotically (using the Coulomb model Refs. 13, 14 predict an effect with the opposite sign and much larger magnitude). Now outside a macroscopic source $\mathcal{S}$ the fluctuation term here should be relatively small compared to $\bar{\Phi}^{2}$, except in rather artificial situations where $\bar{\Phi}$ very nearly vanishes (the net charge and some higher multipoles are exactly zero). The latter are not important in our context, so henceforth I shall drop the fluctuation and write just $\Phi$ for $\bar{\Phi}$.

\section{Natural sources of gravity and $\bar{\psi}$}

First I look at natural sources of $\bar{\psi}$, e.g. the Sun and Earth in the EDB experiments. Unless $\mathcal{S}$ is (almost) exactly neutral, $\Phi$ is dominated by its monopole part. For example, Earth is known to bear a net charge at any time. Thus for a quasispherical source $\Phi \approx Q r^{-1}$ and $|\nabla \bar{\psi}| \approx \kappa^{2} Q^{2} r^{-3}$ at distance $r=|\mathbf{r}|$ from the source's center.

There is a natural bound on $Q$ if the source, a natural large object like Sun or Earth, is to be quiescent: $|Q|<$ $G M m_{p} / e_{0 p}$, where $M$ is $\mathcal{S}$ 's mass, and $m_{p}$ and $e_{0 p}$ the proton's mass and charge, respectively. For if $Q>G M m_{p} / e_{0 p}$, the source's electric field can drive away any free protons formed nearby by, say, cosmic ray ionization of hydrogen even against the pull of gravity, while $\mathcal{S}$ captures the electron and so decreases $Q$. And if $Q<-G M m_{p} / e_{0 p}, \mathcal{S}$ can certainly drive away the free electrons, capture the protons and so decrease $|Q|$. (In the above argument it is important that $e^{2 \psi} \approx 1$ by assumption, so $\mathbf{E} \approx-\nabla \Phi$.) 
In view of the restriction on $Q$,

$$
\begin{aligned}
\kappa|\Phi| & <\left(\frac{1}{4 \pi \alpha}\right)^{1 / 2}\left(\frac{l}{\ell_{P}}\right) \frac{\ell_{P}}{\lambda_{p}} \frac{G M}{c^{2} r} \\
|\nabla \bar{\psi}| & <\frac{1}{4 \pi \alpha c^{2}}\left(\frac{l}{\ell_{P}}\right)^{2}\left(\frac{\ell_{P}}{\lambda_{p}}\right)^{2} \frac{G M}{r^{2}} \frac{G M}{c^{2} r}
\end{aligned}
$$

where $\alpha=e_{0 p}^{2} / \hbar c \approx 0.0073$ and $\lambda_{p}=\hbar / m_{p} c \approx 2 \times 10^{-14} \mathrm{~cm}$ is the proton's Compton length. Obviously $G M / c^{2} r<1$ because the source is not a black hole. It follows that $\kappa|\Phi|<3 \times 10^{-19}\left(l / \ell_{P}\right) \ll 1$, thus verifying the initial assumption. Further, $G M / r^{2} \approx\left|\nabla \phi_{N}\right|$. Thus $|\nabla \bar{\psi}|$ is a fraction $2.2 \times 10^{-37} \zeta^{-1}$ or smaller of the Coulomb model prediction (61). In view of Eq. (62), violations of the WEP reflecting $\mathcal{S}$ 's electric structure show up only at the acceleration fractional level $10^{-40}\left(l / \ell_{P}\right)^{2}$ or smaller. (In light of the conclusions of Secs. III B and IV C, the tiny residual WEP violation here, and similar ones below, may well be artifacts of the macroscopic averaging procedure.) As mentioned already the extant laboratory EDB experiments are sensitive only to acceleration fractional differences at the level of $10^{-13}$. The planned satellite borne STEP experiments are projected to be sensitive only at the level $10^{-18}$.

What if $\mathcal{S}$ is nearly neutral, so that $\Phi$ is dominated by its dipole moment $\mathbf{p}: \Phi \approx \mathbf{p} \cdot \mathbf{r} r^{-3}$. In that case $|\mathbf{p}|$ should be on the order of the radius $R$ of $\mathcal{S}$ times a typical separated charge $\left|Q_{s}\right|$. The magnitudes of the charges $\pm Q_{s}$, which pictorially reside on opposite polar caps of $\mathcal{S}$, are restricted by the same inequality as the monopole charge above. Otherwise free electrons near the negative polar cap would be driven away against gravity and conveyed by the dipole field to the positive cap, thus helping to diminish $|\mathbf{p}|$. Repeating the above argument one finds the expressions for $\kappa|\Phi|$ and $|\nabla \bar{\psi}|$ to be similar to those in Eqs. (64) but each with an extra factor $(R / r)^{2}<1$. Thus $\kappa|\Phi| \ll 1$ is still satisfied, and the failure of the Coulomb model is accentuated. Evidently higher multipoles do not offer a way out of the conclusion that the Coulomb model is very far off the mark. Evidently laboratory and space tests are far from sensitive to violations of the WEP coming from the electric structure of the sources of gravity.

\section{Laboratory sources of gravity and $\bar{\psi}$}

But what if the source $\mathcal{S}$ is not a gravitating body in the dirty interplanetary environment, but rather a mundane body in a clean laboratory where its charge does not immediately get neutralized ? Would the Coulomb model apply then ? An example might be furnished by a lead sphere of radius $R$. Again Eq. (63) tells us that if the sphere holds charge $Q$, then outside it $|\nabla \bar{\psi}| \approx \kappa^{2} Q^{2} r^{-3}<\kappa^{2}\left(Q^{2} / R\right) r^{-2}$. The factor $Q^{2} / R$ is twice the formal Coulomb energy associated with the net charge $Q$. How big can $Q$ be here ? Unless the sphere is in an evacuated cell (which procedure can buy us a few orders of magnitude in the discouraging results below), it can only be charged until its surface electric field reaches the air breakdown level, $\sim 3 \times 10^{4} \mathrm{~V} / \mathrm{cm}=1 \times 10^{2}$ esu. At that point the sphere is at $\Phi \sim 1 \times 10^{2} \mathrm{esu} R$. According to Eq. (9), $\kappa \approx 8.11 \times 10^{-26}$ esu. Thus indeed $\kappa|\Phi| \ll 1$, as assumed. In said state the sphere holds $\sim 1 \times 10^{2}$ esu $R^{2}$ of free charge, and thus twice its macroscopic Coulomb energy is $\sim 1 \times 10^{4} \mathrm{erg} / \mathrm{cm}^{3} R^{3}$. Hence

$$
|\nabla \bar{\psi}|<1 \times 10^{4} \mathrm{erg} / \mathrm{cm}^{3} \kappa^{2} R^{3} r^{-2}
$$

By contrast, in the Coulomb model Eq. (60) would predict

$$
|\nabla \bar{\psi}|=4 \kappa^{2}\left[\zeta\left(4 \pi R^{3} / 3\right) \rho c^{2}\right] r^{-2},
$$

with the square brackets recognizable as the microscopic level Coulomb energy. Being lead $\left(\rho=1.13 \times 10^{1} \mathrm{~g} / \mathrm{cm}^{3}\right.$ and mass number 207.2) the sphere contains $\sim 1.38 \times 10^{23} / \mathrm{cm}^{3} R^{3} \mathrm{~Pb}$ nuclei; each contributes $\sim 795 \mathrm{MeV}$ of $\mathrm{Coulomb}$ energy [29] for a total of $\sim 1.75 \times 10^{20} \mathrm{erg} / \mathrm{cm}^{3} R^{3}$. Thus in the present example the true $|\nabla \bar{\psi}|$, Eq. (65), amounts to a fraction $1.4 \times 10^{-17}$ of the Coulomb model prediction, independent of $R$. In this case also the Coulomb model yields a resounding overestimate. In view of Eq. (62), violations of the WEP for test body motion subject only to the field of a macroscopically charged laboratory sized object are at a fractional level $10^{-23}\left(l / \ell_{P}\right)^{2}$. But this fraction is actually suppressed to $10^{-31}\left(l / \ell_{P}\right)^{2}$ because $\mathcal{S}$ 's gravitational field is diluted by a factor $10^{8}$ by Earth's gravitational field (for this calculation I take $\mathcal{S}$ 's mass to be $10^{6} \mathrm{~g}$ ). Thus the WEP violation considered here is well below the sensitivity of the laboratory EDB experiments. The planned STEP experiments are irrelevant in this context because they do not include their own source of $\bar{\psi}$.

The prospects for WEP violation improve substantially when macroscopically charged sources in the laboratory are replaced by macroscopically polarized ones in Earth orbit (an extension of the STEP experiment which is planned to carry only test objects). A good example is a sphere of radius $R$ made of ferroelectric material. In a ferroelectic there is spontaneous alignment of the molecular electric dipoles, so that a macroscopic polarization vector $\mathbf{P}$ appears. Thus 
in Eq. (63) $\Phi \approx\left(4 \pi R^{3} / 3\right) \mathbf{P} \cdot \mathbf{r} r^{-3}$. A good estimate of the maximum $|\mathbf{P}|$ is one elementary dipole $e_{0 p} d$ per molecular volume $d^{3}$. With $d \sim 10^{-7} \mathrm{~cm}$ this works out to $|\mathbf{P}|<4.8 \times 10^{4}$ esu. Indeed $\mathrm{PbTiO}_{3}$, a ferroelectric with one of the largest measured polarizations, shows $|\mathbf{P}| \sim 1.5 \times 10^{5}$ esu [30]. Given that $r>R$, one finds $\kappa|\Phi|<(4 \pi)^{-1 / 2} \alpha^{1 / 2} l R d^{-2}$. With $R \sim 10^{2} \mathrm{~cm}$ this bound is below $10^{-18}\left(l / \ell_{P}\right)$, small as originally assumed.

Using Eq. (63) one estimates

$$
|\nabla \bar{\psi}| \approx 4 \kappa^{2}(R / r)^{3}\left[\left(4 \pi R^{3} / 3\right) \mathbf{P}^{2}\right] r^{-2}
$$

Eq. (67) exhibits an energy $\mathbf{P}^{2} d^{3}<\left(e_{0 p} d\right)^{2} / d^{3}$ (perhaps tens of eV per molecule) where the Coulomb model's estimate (66) exhibits instead a nuclear Coulomb energy of tens or a few hundred $\mathrm{MeV}$ per nucleus (of which there are a few per molecule). Thus the true $|\nabla \bar{\psi}|$ is a fraction $\left\langle 10^{-7}(R / r)^{3}\right.$ of the Coulomb model prediction. Hence, if it were possible to accurately measure $\mathcal{T}$ 's acceleration at $r \sim 10 R$ from $\mathcal{S}$, the ferroelectric would cause WEP violations at the fractional level $<10^{-16}\left(l / \ell_{P}\right)^{2}$ or smaller. This is within the projected sensitivity of the STEP experiments (which, however, will not carry aloft sources of $\bar{\psi}$ ). But because the electric field near a ferroelectric is very strong, on the order $10^{8}(R / r)^{3} \mathrm{~V} / \mathrm{cm}$ for $\mathrm{PbTiO}_{3}$, it would probably prove necessary to work at considerably smaller $R / r$ to avoid electric perturbations of $\mathcal{T}$. The WEP violation would then most surely become unobservable at the projected STEP sensitivity.

To sum up this subsection, there are no clear cases where extant or planned tests of the WEP are expected to detect violations connected with $\alpha$ variability which originate in the electric structure of matter. But a STEP-like experiment based on a massive ferroelectric source of $\bar{\psi}$ field in Earth's orbit would be a step closer for detection of WEP violation arising from $\alpha$ variability.

\section{Why does the Coulomb model fail so badly?}

Why is the Coulomb model recapitulated in Sec. VA, with its seemingly unassailable logic, so inaccurate ? The answer to this puzzle hinges on a peculiar cancellation which parallels that which facilitated the passage from the complicated Eq. (26) to the simple Eq. (27) in the context of one charged particle. I now explain with the help of macroscopic averaging.

In $\mathcal{S}$ each microscopic particle's mass is subject to Eq. (43). Therefore, macroscopic averaging of Eq. (38) for $\psi$ entails the replacement

$$
\sum_{i} \frac{\partial m_{i} c^{2}}{\partial \psi} \delta^{3}\left(\mathbf{x}-\mathbf{z}_{i}\right) \rightarrow-\frac{1}{\mathcal{V}} \int_{\mathcal{V}} d^{3} x \sum_{i \in \mathcal{V}} \kappa^{-1} e_{0 i} \tan \left(\kappa \Phi\left(\mathbf{z}_{i}\right)\right) \delta^{3}\left(\mathbf{x}-\mathbf{z}_{i}\right) \approx-\frac{1}{\mathcal{V}} \sum_{i \in \mathcal{V}} e_{0 i} \Phi\left(\mathbf{z}_{i}\right)
$$

where the approximation uses the fact (Sec. III C) that at the microscopic level $\kappa|\Phi| \ll 1$, even near a charge, to discard terms of $\mathcal{O}\left(\kappa^{3} \Phi^{3}\right)$. Note that this expression is minus twice the formal microscopic Coulomb energy density in $\mathcal{S}$; the sign is opposite that expected from the logic of the Coulomb model.

Next one needs the macroscopic average of $e^{-2 \psi} \mathbf{E}^{2}$ in $\mathcal{S}$. In the above mentioned approximation Eq. (46), $\mathbf{E}=$ $-e^{2 \psi} \nabla \Phi$, gives with Eq. (45), $e^{2 \psi} \approx 1+\kappa^{2} \Phi^{2}+\cdots$, that

$$
\frac{1}{4 \pi} e^{-2 \psi} \mathbf{E}^{2} \rightarrow \frac{1}{4 \pi \mathcal{V}} \int_{\mathcal{V}} d^{3} x\left[(\nabla \Phi)^{2}+\kappa^{2} \Phi^{2} \mathbf{E}^{2}\right]=\frac{1}{\mathcal{V}} \sum_{i \in \mathcal{V}} e_{0 i} \Phi\left(\mathbf{z}_{i}\right)-\frac{1}{4 \pi \mathcal{V}}\left[\oint_{\partial \mathcal{V}} \Phi \mathbf{E} \cdot d \mathbf{s}-\kappa^{2} \int_{\mathcal{V}} d^{3} x \Phi^{2} \mathbf{E}^{2}\right]
$$

The second form comes from integrating the $(\nabla \Phi)^{2}$ with the help of Gauss's theorem and employing Eq. (40).

Note that the aforementioned microscopic Coulomb energy density term cancels out from the combined contributions (68) and (69). One term that survives is the surface integral over $\Phi \mathbf{E}$. To estimate it recall that if $\partial \mathcal{V}$ is pushed outward somewhat, $\Phi$ on it becomes largely immune to fluctuations from individual charges composing $\mathcal{S}$. Roughly, then, by Gauss's electric law the surface term in Eq. (69) is, $\mathcal{V}^{-1}\langle\Phi\rangle \sum_{i \in \mathcal{V}} e_{0 i}$ where $\langle\Phi\rangle$ is an average of $\Phi$ over the surface. But this expression is on the order of the Coulomb energy per unit volume associated with the net free charge contained in $\mathcal{V}$. And we have seen by example in Sec. VB 2 that this last energy is much smaller than its microscopic counterpart $\mathcal{V}^{-1} \sum_{i \in \mathcal{V}} e_{0 i} \Phi\left(\mathbf{z}_{i}\right)$.

What about the $\kappa^{2} \Phi^{2} \mathbf{E}^{2}$ integral in Eq. (69)? Let $L$ be the smallest microscopic length scale on which $\Phi$ varies, e.g. $10^{-13} \mathrm{~cm}$ if we think of nucleons as the smallest constituents, and perhaps $10^{-17} \mathrm{~cm}$ if quarks and electrons are considered in their stead (already in orthodox quantum electrodynamics, their electric fields are not scale-free Coulombic). Then one expects the maximum $|\Phi|$ to be no larger than a unit charge divided by $L$. Hence on the the average $\kappa^{2} \Phi^{2} \mathbf{E}^{2} / 4 \pi$ is bounded by $\alpha(l / L)^{2}$ times the average microscopic Coulomb energy density $\mathbf{E}^{2} / 4 \pi$. This source of $\bar{\psi}$ is a fraction $<10^{-34}\left(l / \ell_{P}\right)^{2}$ of that assumed in the Coulomb model. It is thus amply clear how the cancellation of Coulomb energy source terms in the equation for $\bar{\psi}$ causes the Coulomb model to grievously overestimate $|\nabla \bar{\psi}|$. 


\section{MAGNETIC STRUCTURE AND WEP}

In ordinary matter, the next largest energy to Coulomb energy is energy of the magnetic dipoles associated with spin and orbital angular momentum of nuclei and electrons. Magnetic energy has so far been ignored here. Eq. (38) is missing the magnetic part of the term $e^{-2 \psi} f_{\mu \nu} f^{\mu \nu}$ in the original scalar equation (16). And in Sec. VC magnetic contributions have been left out of the mass term in Eq. (68). Now that it is clear that Coulomb contributions are well nigh irrelevant as sources of $\bar{\psi}$, it is mandatory to take into account the purely magnetic contributions.

\section{A. Magnetic monopoles: a shortcut to dipoles}

It is a hard task to directly find the analog of the multicharge exact solution (45)-(46) for a collection of magnetic dipoles. But as the example of Sec. VC makes clear, this last solution is essential to derive the correct macroscopic form of the mass term in the source of Eq. (38) for $\psi$. To overcome the problem I first find the analog of Eqs. (45)-(46) for a collection of magnetic monopoles of strengths $g_{0 i}, i=1,2, \cdots 2 N$, and then let pairs of equal and oppositely charged monopoles merge to form magnetic dipoles. The monopoles serve as a calculational crutch here and in the next subsection; they disappear from the final results.

With the notation $\mathbf{B} \equiv\left\{f^{23}, f^{31}, f^{12}\right\}$ I first replace Eqs. (37)-(38) by

$$
\begin{aligned}
& \boldsymbol{\nabla} \times\left(e^{-2 \psi} \mathbf{B}\right)=0 \\
& \boldsymbol{\nabla} \cdot \mathbf{B}=4 \pi \sum_{i} g_{0 i} \delta^{3}\left(\mathbf{x}-\mathbf{z}_{i}\right) \\
& \nabla^{2} \psi=4 \pi \kappa^{2}\left[\sum_{i} \frac{\partial m_{i} c^{2}}{\partial \psi} \delta^{3}\left(\mathbf{x}-\mathbf{z}_{i}\right)-\frac{1}{4 \pi} e^{-2 \psi} \mathbf{B}^{2}\right]
\end{aligned}
$$

Eq. (70) is the space-space component of the Maxwell-type Eq. (14) in a local Lorentz frame and with current and time derivatives set to zero. Eq. (71) does not come from the action (7), but rather generalizes the Gauss law $\boldsymbol{\nabla} \cdot \mathbf{B}=0$ (which follows - also in variable $\alpha$ theory - from the representation of $\mathbf{B}$ as a curl) to the case that there are magnetic monopoles present. This is the argument for not including a factor $e^{-2 \psi}$ in (71). Finally, Eq. (72) is the scalar equation (16) in a local Lorentz frame with electric field and time derivatives dropped.

It is not possible here, in light of Eq. (71), to use a vector potential for $\mathbf{B}$; however, it can be written in terms of a scalar potential (its force on a monopole should be conservative):

$$
\mathbf{B}=-\nabla \Psi
$$

It follows from Eq. (71) and the reasonable boundary condition $\Psi(\mathbf{x}) \rightarrow 0$ as $|\mathbf{x}| \rightarrow \infty$ that

$$
\Psi(\mathbf{x})=\sum_{i} \frac{g_{0 i}}{\left|\mathbf{x}-\mathbf{z}_{i}\right|} .
$$

Eq. (70) now gives $e^{-2 \psi} \nabla \psi \times \nabla \Psi=0$ which uniquely implies that $\psi=\psi(\Psi(\mathbf{x})$ ). Finally, taking the Laplacian of this last relation gives

$$
\begin{aligned}
\nabla^{2} \psi & =\psi^{\prime} \nabla^{2} \Psi+\psi^{\prime \prime}(\nabla \Psi)^{2} \\
& =-4 \pi \psi^{\prime} \sum_{i} g_{0 i} \delta^{3}\left(\mathbf{x}-\mathbf{z}_{i}\right)+\psi^{\prime \prime} \mathbf{B}^{2}
\end{aligned}
$$

Comparison of the second line of Eqs. (75) and Eq. (72) gives

$$
\begin{aligned}
\psi^{\prime \prime} & =-\kappa^{2} e^{-2 \psi} \\
\kappa^{2}\left(\partial m_{i} c^{2} / \partial \psi\right) & =-g_{0 i} \psi^{\prime} \quad \text { at } \quad \mathbf{x}=\mathbf{z}_{i} .
\end{aligned}
$$

The first of these integrates to

$$
\psi^{\prime 2}=\kappa^{2}\left(e^{-2 \psi}+\tilde{\varpi}\right)
$$

with $\tilde{\varpi}$ a constant. All the solutions of this equations may be obtained as in Appendix A:

$$
e^{\psi}=\left\{\begin{array}{cc} 
\pm(\kappa \Psi+\tilde{\chi}) ; & \tilde{\varpi}=0 \\
\pm \tilde{\varpi}^{-1 / 2} \sinh \left(\tilde{\varpi}^{1 / 2} \kappa \Psi+\tilde{\chi}\right) ; & \tilde{\varpi}>0 \\
|\tilde{\varpi}|^{-1 / 2} \cos \left(|\tilde{\varpi}|^{1 / 2} \kappa \Psi+\tilde{\chi}\right) ; & \tilde{\varpi}<0
\end{array}\right.
$$


with $\tilde{\chi}$ a second integration constant.

Because we made no guesses or ansatze along the way to Eqs. (79), these last together with Eq. (73) and (74) must exhaust the solutions of Eqs. (70)-(72) with boundary conditions $\Psi(\mathbf{x}) \rightarrow 0$ as $|\mathbf{x}| \rightarrow 0$. In this connection it is appropriate to remark that the substitution $e^{2 \psi} \mathbf{B} \mapsto \mathbf{E}$ followed by $\psi \mapsto-\psi$ and $g_{0 i} \mapsto e_{0 i}$, transform the magnetic solution (73)-(74) together with each of the five variants in Eq. (79) into one of the five variants of the electric solution (41)-(42) of Sec. IV A. A similar transformation maps each electric solution back onto a magnetic solution. The one-to-one correspondence between magnetic and electric solutions reflects duality symmetry of the $\alpha$ variability framework. Since all time-independent magnetic solutions have been found, the principle of duality assures us that all time-independent electric solutions have been found as well.

Also by duality, the magnetic solution with $\tilde{\varpi}<0$, which is dual to the physical electric solution $(\varpi<0)$, must be regarded as the physical choice. In fact, if invoking by now well worn arguments, we set $\tilde{\varpi}=-1$ and $\tilde{\chi}=0$, that solution is consistent with the reasonable boundary condition $\psi \rightarrow 0$ as $|\mathbf{x}| \rightarrow \infty$, whereas the solutions with $\tilde{\varpi} \geq 0$ cannot be made so. Thus the unique physical solution for a collection of magnetic monopoles at rest is given by Eqs. (73) together with

$$
\begin{aligned}
e^{\psi} & =\cos (\kappa \Psi) \\
\mathbf{B} & =-\nabla \Psi .
\end{aligned}
$$

\section{B. Magnetic dipoles as source of $\bar{\psi}$}

Returning to our main subject, magnetic dipole energy, I imagine that the monopole labeled by $i+N$ has magnetic charge $-g_{0 i}$, and denote the vector from monopole $i+N$ to monopole $i$ by $\mathbf{d}$. Then if as the pair are allowed to approach each other adiabatically, $\left|g_{0 i}\right|$ is made to grow in such a way that $g_{0 i} \mathbf{d} \rightarrow \boldsymbol{\mu} \neq 0$ in the limit, a point magnetic dipole is formed. It possesses magnetic moment $\boldsymbol{\mu}$ and may be labeled by $i$. I still refer to its position as $\mathbf{z}_{i}$.

The total magnetic potential is

$$
\begin{aligned}
\Psi & =\lim _{|\mathbf{d}| \rightarrow 0} \sum_{i=1}^{N}\left[\frac{g_{0 i}}{\left|\mathbf{x}-\mathbf{z}_{i}-\mathbf{d} / 2\right|}+\frac{-g_{0 i}}{\left|\mathbf{x}-\mathbf{z}_{i}+\mathbf{d} / 2\right|}\right] \\
& =\sum_{i=1}^{N} \frac{\boldsymbol{\mu}_{i} \cdot\left(\mathbf{x}-\mathbf{z}_{i}\right)}{\left|\mathbf{x}-\mathbf{z}_{i}\right|^{3}} .
\end{aligned}
$$

Thus $e^{\psi}$ is explicitly known from Eq. (81). Performing the gradient in Eq. (81) gives for the field of the dipole,

$$
\mathbf{B}=\sum_{i=1}^{N}\left[\frac{3 \boldsymbol{\mu} \cdot\left(\mathbf{x}-\mathbf{z}_{i}\right)\left(\mathbf{x}-\mathbf{z}_{i}\right)}{\left|\mathbf{x}-\mathbf{z}_{i}\right|^{5}}-\frac{\boldsymbol{\mu}}{\left|\mathbf{x}-\mathbf{z}_{i}\right|^{3}}\right] .
$$

This coincides (apart from the singularities at $\mathbf{x}=\mathbf{z}_{i}$ ) with the Maxwellian expression for the field of an array of magnetic dipoles derived from the vector potential [28]. It seems reasonable that were magnetic dipoles to be represented by tiny current loops, the field would still be (83). Eqs. (80)-(82) thus represent the multimagnetic dipoles solution within the framework.

What is the source term in the equation for $\psi$ for matter made exclusively of magnetic dipoles ? From Eq. (80) it follows that

$$
\nabla^{2} \psi=\kappa \tan (\kappa \Psi) \boldsymbol{\nabla} \cdot \mathbf{B}-\kappa^{2} \sec ^{2}(\kappa \Psi) \mathbf{B}^{2}
$$

It is easy to verify, for example by applying Gauss' theorem to Eq. (83), that $\boldsymbol{\nabla} \cdot \mathbf{B}$ vanishes everywhere, and has no $\delta$-function singularity at the position of a dipole. Since $\sec ^{2}(\kappa \Psi)=e^{-2 \psi}$, comparison of the last result with Eq. (72) shows that the mass-dependent source term is absent for magnetic dipoles. It is easy to get a wrong answer in this respect, for example, by trying to combine the mass-dependent terms of the positive and negative poles before taking the limit $|\mathbf{d}| \rightarrow 0$. Since the mass-dependent source term would arise naturally from the action, the conclusion must be that the mass of a pointlike magnetic dipole, unlike that of a pointlike charge, does not depend on $\psi$.

An immediate consequence is that a magnetic dipole moment in an exterior electromagnetic field is not subject to an anomalous force of the sort appearing for an electric charge, cf. Eq. (26). The only force would be the usual $(\boldsymbol{\mu} \cdot \boldsymbol{\nabla}) \mathbf{B}$ obtainable in our context by combining the forces on two magnetic poles. All this means that no violations of the WEP are expected in the motion of matter with pure magnetic dipole structure; it is unnecessary in this connection to carry out the analog of the calculation in Secs. IV B and IV C. 
But does a source $\mathcal{S}$ with purely magnetic dipole structure cause WEP violations in the motion of a test body $\mathcal{T}$ made up of electric charges? In analogy with the argument in Sec. V B it follows from Eq. (80) that

$$
\bar{\psi} \approx-\frac{1}{2} \kappa^{2} \overline{\Psi^{2}}=-\frac{1}{2} \kappa^{2} \bar{\Psi}^{2}-\frac{1}{2} \kappa^{2} \overline{(\Psi-\bar{\Psi})^{2}}
$$

Contrary to what happens in the presence of a source with electric structure, cf. Sec. VB, here the physical $\alpha$ has its value slightly depressed in relation to the asymptotic value. As in Sec. VB, outside a macroscopic source $\mathcal{S}$, the fluctuation term should be relatively small compared to $\bar{\Psi}^{2}$, except in rather artificial situations where $\bar{\Psi}$ vanishes $(\mathcal{S}$ has no net magnetic moment). The latter are not important in our context, so henceforth I shall drop the fluctuation and write just $\Psi$ for $\bar{\Psi}$.

Supposing $\mathcal{S}$ to be a sphere with uniform magnetization $\mathbf{M}, \Psi$ in Eq. (82) is well approximated at distance $r \gg R$ from the sphere's center by $\left(4 \pi R^{3} / 3\right) \mathbf{M} \cdot \mathbf{r} r^{-3}$. Then

$$
|\nabla \bar{\psi}| \approx 4 \kappa^{2}(R / r)^{3}\left[\left(4 \pi R^{3} / 3\right) \mathbf{M}^{2}\right] r^{-2}
$$

If Earth is the source $\mathcal{S}$, its crudely dipolar magnetic field ( $\sim 0.25 \mathrm{G}$ at the magnetic poles) allows its representation by a uniformly magnetized sphere with $|\mathbf{M}| \sim 3 \times 10^{-2}$ cgs [28]. The energy represented by the square brackets in Eq. (86), $\sim 9.7 \times 10^{23} \mathrm{erg}$, is a fraction $\sim 10^{-22}$ of the corresponding factor in Eq. (66) for the Coulomb model (I take $\zeta=10^{-3}$ for Earth). Hence Earth, by virtue of its magnetic structure, causes WEP violations at the fractional level $10^{-28}(l / \ell)^{2}$, well beyond anything measurable in the foreseeable future.

Another example of $\mathcal{S}$ is furnished by a ferromagnetic sphere. Iron, one of the ferromagnets with the highest saturation magnetization, can reach $|\mathbf{M}| \approx 1.7 \times 10^{3} \mathrm{cgs}$ [30]. The energy in the square brackets in Eq. (86) is then $\approx 1.2 \times 10^{7} \mathrm{erg} / \mathrm{cm}^{3} R^{3}$. Being iron $\left(\rho=7.87 \mathrm{~g} / \mathrm{cm}^{3}\right.$ and mass number 55.84$)$ the sphere contains $\sim 3.55 \times 10^{23} / \mathrm{cm}^{3} R^{3}$ Fe nuclei; each contributes $\sim 125 \mathrm{MeV}$ of Coulomb energy [29] for a total of $\sim 7.11 \times 10^{19} \mathrm{erg} / \mathrm{cm}^{3} R^{3}$. Thus the true $|\nabla \bar{\psi}|$, Eq. (86), amounts to a fraction $1.7 \times 10^{-13}(R / r)^{3}$ of the Coulomb model prediction. It follows that a ferromagnetic source of $\bar{\psi}$ in orbit would cause WEP violations at a fractional level well below $10^{-19}$. This is beyond the projected STEP sensitivity (and, of course, the STEP experiment will not carry the equivalent of $\mathcal{S}$ ).

To be sure the above procedure is a bit cavalier. The exact solution (80)-(82) which forms the ultimate basis of the calculation contains no electric charges, while Earth and iron have plenty of them. But this defect is not serious. Let $\psi \equiv \psi_{\mathrm{e}}(\Phi)+\psi_{\mathrm{m}}(\Psi)$ with $\psi_{\mathrm{m}}$ the function $\psi(\Psi)$ in Eq. (80) and $\psi_{\mathrm{e}}$ the $\psi(\Phi)$ defined by Eq. (45) for the collection of electric charges. Then the electric field $\mathbf{E}$ in Eq. (46) solves the Gauss equation (37), to within a relative correction of $\mathcal{O}\left(\psi_{\mathrm{m}}\right)$, and is a gradient. Likewise, the magnetic field $\mathbf{B}$ [Eq. (83)] deriving from $\Psi$ in Eq. (82) satisfies, to within a fractional correction of $\mathcal{O}\left(\psi_{\mathrm{e}}\right)$, the Ampere-like Eq. (70), and is also divergence free (Gauss's magnetic equation).

Further, adding Eq. (38) for $\psi_{\mathrm{e}}$ to Eq. (72) (without the mass term) for $\psi_{\mathrm{m}}$ gives, to within fractional corrections of $\mathcal{O}\left(\psi_{\mathrm{e}}\right)$ and $\mathcal{O}\left(\psi_{\mathrm{m}}\right)$, the correct equation for the full $\psi$ relevant for a collection of charges and magnetic dipoles, cf. Eq. (16):

$$
\nabla^{2} \psi=4 \pi \kappa^{2}\left[\sum_{i} \frac{\partial m_{i} c^{2}}{\partial \psi} \delta^{3}\left(\mathbf{x}-\mathbf{z}_{i}\right)+\frac{1}{4 \pi} e^{-2 \psi}\left(\mathbf{E}^{2}-\mathbf{B}^{2}\right)\right]
$$

The sum here extends only over electric charges. Plainly the electric and magnetic contributions to $\psi$ are additive (because $\left|\psi_{\mathrm{e}}\right| \ll 1$ and $\left|\psi_{\mathrm{m}}\right| \ll 1$ ). Thus the correct $\bar{\psi}$ to use for real matter (charges plus magnetic dipoles) is, not that in Eq. (85), but rather

$$
\bar{\psi} \approx \frac{1}{2} \kappa^{2}\left(\bar{\Phi}^{2}-\bar{\Psi}^{2}\right) .
$$

In view of this composition rule, the results of Sec. VIB that WEP violations originating from $\psi_{\mathrm{m}}$ will be unobservable for the foreseeable future, together with those of Sec. V B that effects coming from $\psi_{\mathrm{e}}$ have the same status, show there are no clear cases where extant or planned tests of the WEP are expected to detect violations connected with $\alpha$ variability. Still outstanding is the question whether motion of charges or dipoles in matter - totally ignored in this paper - could provide a loophole from this conclusion.

\section{RESOLVING A COSMOLOGICAL CONUNDRUM}

The cosmological evolution of $\alpha$ according to the framework is influenced by the nature of $\psi$ 's source and by a certain integration constant $t_{C}$ (a cosmological time scale) [9]. If the source is described with the Coulomb model applied to ordinary matter of cosmological abundances, and the appealing choice $t_{C}=0$ is made, then $\alpha$ is predicted 
to decrease throughout the matter dominated era $[9,11,14]$. The claimed cosmological rise of $\alpha[1]$ thus led Olive and Pospelov [11] and Barrow, Sandvik and Magueijo [16] to conclude that the scalar field is coupled mostly to dark matter with a coupling opposite in sign to the naive one. To quote Ref. [16], "... the dark matter constituents have to have a high magnetostatic energy content (one possible contender would be superconducting cosmic strings which have $\zeta \approx-1$ )"; as clear from Eq. (87), magnetic fields are sources of $\psi$ with effectively negative $\zeta$.

Arguably the mentioned resolution of the cosmological conundrum amounts to trading one cosmic mystery (growing $\alpha$ ) for another (dark matter with especially unusual electromagnetic properties). A much more natural solution is offered by the realization (see Sec. VC) that the Coulomb energy cancels from the source term of $\psi$ 's equation. This should also be true for cosmological baryonic matter because one can think of each small region of the cosmological medium as being at rest in a local Lorentz frame. Consequently, the source of $\psi$ 's cosmological evolution is principally the $\mathbf{B}^{2}$ term contributed by matter's magnetic dipoles to the cosmological version of Eq. (87). [By Sec. VIB the mass term in Eq. (16) is absent for magnetic dipoles, thermal radiation does not contribute at all because $\mathbf{E}^{2}-\mathbf{B}^{2}$ vanishes for electromagnetic radiation [9], and cosmological magnetic fields are too weak to make a difference.] Thus the switch in sign of the source term required by the claimed cosmological increase of $\alpha$ comes about automatically by considering the magnetostatic energy of baryonic cosmological matter. As I show now, the rate at which $\alpha$ grows also comes out of the right order without special assumptions.

For cosmological $\alpha$ variability the interesting field is $\bar{\psi}$. As already discussed, it will obey an equation like (16) but with the source averaged over a macroscopic region. This average magnetic energy density is easiest evaluated by first computing the magnetic energy associated with a given mass of cosmological matter.

Now in baryonic matter the magnetic energy is principally tied to the protons' and electrons' magnetic moments $\left({ }^{4} \mathrm{He}\right.$ nuclei have no magnetic moment). A lower bound on (and a passably good estimate for) the magnetic energy $E_{p}$ of the proton should result from regarding it as a perfect magnetic dipole and ignoring the energy interior to the proton radius $R_{p}$. Integrating $\mathbf{B}^{2} / 8 \pi$ (cf. the electric energy in Appendix B) with $\mathbf{B}$ in Eq. (83) over the space outside $R_{p}$ gives $E_{p} \gtrsim \mu^{2} / 3 R_{p}{ }^{3}$. Now for the proton $\mu=2.98 \times e \hbar / 2 m_{p} c$ [29], whereas $R_{p} \approx 5.7\left(\hbar / m_{p} c\right)$. Thus $E_{p} \gtrsim 2.54 \times 10^{-5} m_{p} c^{2}$. For the electron $\mu=e \hbar / 2 m_{e} c$. In view of the Zitterbewegung phenomenon is seems reasonable to integrate the magnetic energy of the dipole field only down to the Compton length $\hbar / m_{e} c$. Doing this as in the proton calculation gives $E_{e} \gtrsim 6.1 \times 10^{-4} m_{e} c^{2}$. Other magnetic energies, e.g. those connected with spin-orbit and hyperfine splittings, are small on the Bohr scale $\alpha^{2} m_{e} c^{2}$, and thus negligible here.

In the context of a matter-dominated Robertson-Walker expanding model (with no cosmological constant and neglecting $\psi$ 's effect on the expansion as appropriate at late cosmological time), one can use Eq. (31) of Ref. [9] for the rate of change of $\alpha$ :

$$
\dot{\alpha} / \alpha=-(3 / 4 \pi)\left(l / \ell_{P}\right)^{2} \zeta \Omega_{b} H^{2} t
$$

Here $H$ is the Hubble "constant" at cosmological time $t, \Omega_{b}$ the baryon density parameter and $|\zeta|$ the fraction of the cosmic baryon mass density $\rho_{b}$ which acts as source of $\bar{\psi}$ (here $\zeta<0$ ). Now $23 \%$ of $\rho_{b}$ consists of ${ }^{4}$ He. From the above results the magnetic energy density from helium's two electrons, and the protons and electrons from hydrogen, when appropriately weighted by their cosmic abundances, is $\mathcal{E}_{m} \gtrsim 1.98 \times 10^{-5} \rho_{b} c^{2}$, meaning that $\zeta \lesssim-1.98 \times 10^{-5}$. With $H t=2 / 3$ (matter domination) and today's favorite value $\Omega_{b}=0.03$, Eq. (89) predicts

$$
\dot{\alpha} / \alpha \gtrsim 6 \times 10^{-8}\left(l / \ell_{P}\right)^{2} t^{-1} .
$$

As advertised, $\alpha$ is predicted to increase with time. In order for its overall fractional change over the last $10^{10}$ y to match the observed $0.7 \times 10^{-5}$, it is necessary that $l \lesssim 10 \ell_{P}$. This is a reasonable value consistent with the framework's assumptions and with all tests of the WEP (see Secs. V B and VIB). Hence nothing but baryonic matter is necessary to explain the claimed cosmological sense and rate of $\alpha$ variation. Were this last to be ruled out by future observations, one would have to conclude that $l$ is smaller than mentioned. Within the spirit of the framework an $l$ much below $\ell_{P}$ is unacceptable, and so one can risk a flat prediction that in recent epochs $t \dot{\alpha} / \alpha>10^{-8}$. Observational exclusion of this bound would be tantamount to certifying that standard Maxwellian electrodynamics (the case $l \equiv 0$ ) is the exact classical description of electromagnetism.

\section{CONCLUSIONS AND CAVEATS}

The issue of $\alpha$ variability has long been connected with the possibility of weak equivalence principle violations coming from the classical Coulomb energy contribution to particle masses. The central conclusion of this paper is that in the general framework for $\alpha$ variability described in Ref. [9], a compensating mechanism exists which prevents such violations from being measurable, at least in the foreseeable future. The treatment has been classical or tree level. Quantum considerations raise a potential problem for this kind of theory. 
Banks, Dine and Douglas [31] have argued that in a theory where a scalar field couples to the electromagnetic scalar $f_{\mu \nu} f^{\mu \nu}$, as in the action (7), the residual vacuum energy of matter fields is very much larger than the observed cosmological constant unless the parameters are finely tuned. Recall that even with no scalar coupling the vacuum energy is known to be formally very large. But it is widely believed that some as yet ill understood mechanism nearly cancels it. Ref. [31] points out that because of the variation of the scalar field required for the suggested explanation of cosmological $\alpha$ variation to work, this cancellation can be successful only over a very short interval of cosmological time, with the residual vacuum energy becoming intolerably large earlier and thereafter.

In light of this argument, can a theory like the framework used here be taken seriously ? One has to recall that the aforesaid criticism presupposes the existence of a cancellation of the vacuum energy by a mechanism whose nature is not generally agreed upon today. One can well imagine that when that mystery is lifted, a resolution for the problem of large variation of the residual vacuum energy with the scalar field might also become apparent. At any rate, it is premature to draw any final conclusions at this stage since the problem remarked upon in Ref. 31 is an integral part of the unsolved mystery of the small cosmological constant.

\section{Acknowledgments}

I am indebted to Cristophe Grojean, John Moffat, Jonathan Oppenheim and especially to Gia Dvali and Matias Zaldarriaga for remarks and criticisms which sharpened my understanding of the issues discussed here. This research was supported by grant No. 129/00-1 of the Israel Science Foundation.

\section{APPENDIX A: SOLUTIONS FOR $\psi$}

For $\varpi=0$ take a square root of Eq. (24) to get

$$
e^{-\psi} \psi^{\prime}= \pm \kappa
$$

which may immediately be integrated to

$$
e^{\psi}=\mp(\kappa V+\chi)^{-1}
$$

with $\chi$ a constant.

For $\varpi \neq 0$ set $\psi=\ln y+\frac{1}{2} \ln |\varpi|$. Then after taking a square root, Eq. (24) transforms into

$$
\frac{d y}{y \sqrt{y^{2}+1 \cdot \operatorname{sgn}(\varpi)}}= \pm|\varpi|^{1 / 2} \kappa d V
$$

In the case $\varpi>0$ the l.h.s. of this last equation is the differential of $\operatorname{arccsch}(y)$ [the upper (lower) signs corresponding to negative (positive) $y$ ]. Thus

$$
y= \pm \operatorname{csch}\left(\varpi^{1 / 2} \kappa V+\chi\right) .
$$

In the case $\varpi<0$ the l.h.s. of Eq. (A3) is the differential of $\operatorname{arcsec}(y)$ for either sign of $y$. Thus $\operatorname{since} \sec ( \pm y)=\sec (y)$,

$$
e^{\psi}=\left\{\begin{array}{cl} 
\pm(\kappa \Phi+\chi)^{-1} ; & \varpi=0, \\
\pm \sqrt{ } \varpi \operatorname{csch}(\sqrt{ } \varpi \kappa \Phi+\chi) ; & \varpi>0, \\
\sqrt{ }|\varpi| \sec (\sqrt{ }|\varpi| \kappa \Phi+\chi) ; & \varpi<0 .
\end{array}\right.
$$

\section{APPENDIX B: APPLICABILITY OF THE REISSNER-NORDSTRÖM METRIC}

Here I focus on an elementary charge. To assess the corrections to the metric coming from the scalar field I write down the energy-momentum tensor contributions from $\psi$ and $f^{\mu \nu}$ in curved spacetime in accordance with Eqs. (6)-(7):

$$
\begin{aligned}
T_{(\psi)}{ }^{\mu}{ }_{\nu} & =\frac{1}{4 \pi \kappa^{2}}\left(\psi_{,}{ }^{\mu} \psi_{, \nu}-\frac{1}{2} g^{\mu}{ }_{\nu} \psi_{,}{ }^{\alpha} \psi_{, \alpha}\right) \\
T_{(f)}{ }^{\mu}{ }_{\nu} & =\frac{e^{-2 \psi}}{4 \pi}\left(f^{\mu \alpha} f_{\nu \alpha}-\frac{1}{4} g^{\mu}{ }_{\nu} f^{\alpha \beta} f_{\alpha \beta}\right)
\end{aligned}
$$


In either tensor energy densities and stresses have the same magnitudes for a spherical static solution for which necessarily $\psi_{, r}$ and $f^{t r}$ are the only surviving components of $\psi_{, \mu}$ and $f^{\mu \nu}$, respectively). In the weak gravity region $\left(r \gg R\right.$ ) one may use Eq. (20), the fact that $\nabla \psi=\psi^{\prime} \nabla \Phi$ and Eq. (33) (with $\chi=0$ ) to get for the said solution

$$
\frac{\left|T_{(\psi)}{ }^{\mu}{ }_{\nu}\right|}{\left|T_{(f)}{ }_{\nu}\right|}=\kappa^{-2} \psi^{\prime 2} e^{2 \psi}=\sec ^{2} \kappa \Phi \tan ^{2} \kappa \Phi
$$

$T_{(\psi)}{ }_{\nu}{ }_{\nu}$ is thus negligible compared to $T_{(f)}{ }^{\mu}{ }_{\nu}$ all the way in to a radius where $|\Phi|$ is no longer small compared to $\pi^{3 / 2}(\hbar c)^{1 / 2} l^{-1}$. According to Sec. III C the said radius represents the smallest possible extension of the charge. Hence, unless the charge is as compact as permitted, one can consistently neglect $T_{(\psi)}{ }_{\nu}{ }_{\nu}$ in the above equations over the entire charge's exterior, and can do so over most of the exterior in the compact charge case.

A look at Eq. (21) shows that $f_{t r}$ here is $e^{2 \psi}$ times its Maxwellian counterpart $c \nabla \Phi$. Hence, $T_{(f)}{ }^{\mu}{ }_{\nu}$ here differs from the pure Maxwellian energy momentum tensor also by a factor $e^{2 \psi}=\sec ^{2} \kappa \Phi$, which is very close to unity all the way in to the radius mentioned above. Thus the Maxwellian energy momentum tensor replaces the full $T^{\mu}{ }_{\nu}$ throughout the whole exterior of a not maximally compact charge, and throughout most of it for a compact charge. As a consequence the Reissner-Nordström metric (35) is rather accurate outside the charge.

\section{APPENDIX C: THE MASS FUNCTION}

What can be said about spatial variation of masses of electrically charged particles through their dependence on $\psi$ ? For simplicity of notation I focus on that of charge 1 in the cluster discussed in Sec. IV B. I first rewrite Eq. (43) with the help of Eq. (45) as

$$
\left(\partial m_{1} c^{2} / \partial \Phi\right)=-e_{01} \tan ^{2}(\kappa \Phi)
$$

Evidently $\Phi$ here means the value of the potential (39) evaluated at the position of charge 1, and of course this potential is large there (not infinite, though, since as mentioned in Sec. IV B, charges cannot be exact points in this framework). The equation refers to the change of $m_{1}$ resulting from the local change of $\Phi$ which might be due, for example, to the other charges being moved around (adiabatically).

Proceeding purely formally I use the fact that $d \tan x / d x=1+\tan ^{2} x$ to integrate Eq. (C1):

$$
m_{1} c^{2}=m_{01} c^{2}+e_{01}\left[\Phi-\kappa^{-1} \tan (\kappa \Phi)\right]
$$

Here $m_{01}$ is a constant of integration. It is clear from this that $m_{1}$ is invariant under conjugation of all charges, as would be expected by $\mathrm{C}$ invariance of the electromagnetic interaction; although the framework's assumptions require only T invariance, the theory's action (6)-(8) is actually $\mathrm{C}$ invariant as well. According to Eq. $(33), \kappa \Phi=\operatorname{arcsec} e^{\psi}$; since $\tan ^{2}(\kappa \Phi)=\sec ^{2}(\kappa \Phi)-1$ one has

$$
m_{1} c^{2}=m_{01} c^{2}+\kappa^{-1} e_{01}\left(\operatorname{arcsec} e^{\psi}-\sqrt{e^{2 \psi}-1}\right) .
$$

The appropriateness of choosing the shown sign of the square root is verified by differentiating $m_{1}(\psi)$ and comparing the result with Eqs. (43) and (33). The opposite sign is excluded on this ground.

As anticipated, the space-dependent contribution to $m_{1}$ is not proportional to $e_{0 i}{ }^{2} e^{2 \psi}$ as would be assumed in the Coulomb model.

Unfortunately, Eqs. (C2) and (C3) are not immediately useful; they contain a contribution from the self potential of particle 1 or from its self $\psi$ field. If there were no other charges in the universe, one would expect $m_{1}$ to subsume these contributions. Absorbing the self terms of Eq. (C2) into $m_{01}$ gives

$$
m_{1} c^{2}=m_{01} c^{2}+e_{01}\left\{\Phi_{i>1}-\kappa^{-1}\left[\tan (\kappa \Phi)-\tan \left(\kappa \Phi_{i=1}\right)\right]\right\}
$$

The identity $(\tan x-\tan y) \cos x \cos y=\sin (x-y)$ allows us to rewrite this as

$$
m_{1} c^{2}=m_{01} c^{2}+e_{01}\left[\Phi_{i>1}-\kappa^{-1} A \sin \left(\kappa \Phi_{i>1}\right)\right]
$$

where $A \equiv \sec \left(\kappa \Phi_{i=1}\right) \sec (\kappa \Phi)$ is evaluated at particle 1's center.

Quite generically $\kappa\left|\Phi_{i>1}\right| \ll 1$. This merely requires that the overall size $\tilde{R}$ of the cluster of charges $i=2,3, \cdots$, and its overall charge sans $e_{01}, \tilde{Q}$, satisfy

$$
\tilde{R} \gg \pi^{-3 / 2}\left(\tilde{Q}^{2} / \hbar c\right)^{1 / 2}\left(l / \ell_{P}\right) \ell_{P}
$$


which is easily met by nucleons, nuclei, ions and macroscopic charged objects. One expects charge 1 to be relatively more compact so that $\left|\Phi_{i=1}\right| \gg\left|\Phi_{i>1}\right|$. In this case Taylor expanding $A$ to first order in $\kappa \Phi_{i>1}$ as well as $\sin \left(\kappa \Phi_{i>1}\right)$ to second order converts Eq. (C5) into

$$
m_{1} c^{2}=m_{01} c^{2}-e_{01} \sec ^{2}\left(\kappa \Phi_{i=1}\right) \times\left[\sin ^{2}\left(\kappa \Phi_{i=1}\right) \Phi_{i>1}+\kappa \tan \left(\kappa \Phi_{i=1}\right) \Phi_{i>1}{ }^{2}+\mathcal{O}\left(\Phi_{i>1}{ }^{3}\right)\right]
$$

With the possible exclusion of the "point" leptons and quarks, even elementary particles are much more extended than the bound (34); thus $\kappa\left|\Phi_{i=1}\right| \ll 1$. Hence the variable part of $m_{1}$ is generally very small compared to the Coulomb potential energy of that charge in the cluster, $e_{01} \Phi_{i>1}$, and of opposite sign.

[1] J. K. Webb, V. V. Flambaum, C. W. Churchill, M. J. Drinkwater and J. D. Barrow, Phys. Rev. Lett. 82, 884 (1999); J. K. Webb, M. T. Murphy, V. V. Flambaum, V. A. Dzuba, J. D. Barrow, C. W. Churchill, J. X. Prochaska and A. M. Wolfe, Phys. Rev. Lett. 87, 091301 (2001).

[2] P. Jordan, Naturwiss. 25, 513 (1937); Z. f. Physik 113, 660 (1939).

[3] E. Teller, Phys. Rev. 73, 801 (1948).

[4] K. P. Stanyukovich, Dokladii Akad. Nauk SSSR 147, 1348 (1962) [Sov. Phys. Doklady 7, 1150 (1963)].

[5] G. Gamow, Phys. Rev. Lett. 19, 759, 913 (1967).

[6] R. H. Dicke, The Theoretical Significance of Experimental Relativity (Gordon and Breach, New York 1965).

[7] R. H. Dicke, Science 129, 621 (1959).

[8] J. P. Uzan, hep-ph/0205340.

[9] J. D. Bekenstein, Phys. Rev. D 25, 1527 (1982).

[10] M. Livio and M. Stiavelli, Astrophys. J. Lett. 507, L13 (1998).

[11] K. A. Olive and M. Pospelov, Phys. Rev. D 65, 085044 (2002).

[12] G. Dvali and M. Zaldarriaga, Phys. Rev. Lett. 88, 091303 (2002).

[13] J. Magueijo, J. D. Barrow and H. B. Sandvik, astro-ph/0202374.

[14] H. B. Sandvik, J. D. Barrow and J. Magueijo, Phys. Rev. Lett. 88, 031302 (2002).

[15] J. D. Barrow, H. B. Sandvik and J. Magueijo, Phys. Rev. D65, 063504 (2002).

[16] J. D. Barrow, H. B. Sandvik and J. Magueijo, Phys. Rev. D 65123501 (2002).

[17] J. D. Barrow and C. O'Toole, astro-ph/9904116.

[18] J. D. Barrow and D. F. Mota, gr-qc/0207012.

[19] S. J. Landau, P. D. Sisterna and H. Vucetich, Phys. Rev. D 63, 081303(R) (2001).

[20] J. W. Moffat, Found. Phys. 23, 411 (1993) and Int. J. Mod. Phys, D 2, 351 (1993).

[21] J. Magueijo, Phys. Rev. D 62, 103521 (2000).

[22] J. D. Bekenstein, Comm. Astrophys. 8, 89 (1979).

[23] M. J. Duff, hep-th/0208093.

[24] J. W. Moffat, hep-th/0208109.

[25] G. Dvali and M. Zaldarriaga, unpublished.

[26] Z. Chacko, C. Grojean and M. Perelstein, hep-ph/0204142.

[27] J. D. Bekenstein and M. Milgrom, Astroph. Journ. 286, 7 (1984).

[28] J. D. Jackson, Classical Electrodynamics (Wiley, New York 1962).

[29] E. Segre, Nuclei and Particles (Benjamin, New York 1965).

[30] C. Kittel, Introduction to Solid State Physics, 3rd edition (Wiley, New York 1966).

[31] T. Banks, M. Dine and M. Douglas, Phys. Rev. Lett. 88, 131301 (2002). 